\title{
Floristic survey in an Atlantic Forest remnant in the Recôncavo da Bahia, Bahia State, Brazil
}

\author{
Douglas Machado Moreira ${ }^{1,2}$, (D) Grênivel Costa ${ }^{2}$, (D) Josival Santos Souza² and (D) Lidyanne Yuriko Saleme Aona ${ }^{2,3}$
}

Submetido: 20.05.2019; aceito: 28.02.2020

How to cite: Moreira, D.M., Costa, G., Souza, J.S. \& Aona, L.Y.S. 2020. Floristic survey in an Atlantic Forest remnant in the Recôncavo da Bahia, Bahia State, Brazil. Hoehnea 47: e572019. http://dx.doi.org/10.1590/2236-8906-57/2019

RESUMO - (Levantamento florístico em remanescente de Mata Atlântica no Recôncavo da Bahia, Estado da Bahia, Brasil). O presente trabalho apresenta a lista de Angiospermas ocorrentes em um fragmento de Floresta Atlântica localizado no Recôncavo da Bahia. O estudo foi desenvolvido na Serra da Copioba, município de São Felipe, Estado da Bahia, Brasil. Expedições botânicas foram realizadas à área de estudo entre os anos de 2016 e 2017. As coletas foram realizadas a partir de trilhas por toda a região da Serra da Copioba. Todo o material coletado foi depositado no Herbário do Recôncavo da Bahia (HURB). Foram registradas 306 espécies, das quais 20,46\% são endêmicas da Floresta Atlântca e 4,25\% são endêmicas do Estado da Bahia. Os índices de endemismo somados a ocorrência exclusiva de plantas fazem com que a Serra da Copioba possa ser considerada um importante remanecente de Floresta Atlântica da região.

Palavras-chave: Angiospermas, checklist, florística

ABSTRACT - (Floristic survey in an Atlantic Forest remnant in the Recôncavo da Bahia, Bahia State, Brazil). This work presents a list of the Angiosperms that occurs in a fragment of the Atlantic Forest, Recôncavo da Bahia region. The present study was carried out in the Serra da Copioba in the municipality of São Felipe, Bahia State, Brazil. Botanical expeditions were carried out in the studied area between the years 2016 and 2017. The collections were performed in trails throughout the region of the Serra da Copioba. The specimens were housed in the Herbarium of the Recôncavo da Bahia (HURB). We found 306 species. The rates of endemism registered by this study are $20.46 \%$ for species occurring exclusively in the Atlantic Forest and 4.25\% are endemic to Bahia State. The good indexes of endemism added to the exclusive occurrence of plants make the Copioba Mountain an important remnant of the Atlantic Forest of the region.

Keywords: Angiosperms, checklist, floristics

\section{Introduction}

The Atlantic Forest is a phytogeographical domain formed by a set different vegetations in the Brazilian coast that originally occupied about $1,315,460 \mathrm{~km}^{2}$, however, currently only $12.5 \%$ of this area is preserved and distributed in small and threatened fragments (SOS Mata Atlântica 2018). The Atlantic Forest has been explored since the colonization of Brazil and its devastation has been intensified with the development of cities, road construction, exploitation of natural resources and agricultural expansion from the coast (Morellato \& Haddad 2000, Joly et al. 2014).
The Atlantic Forest is considered one of the most threatened hotspots in the world, besides being a great center of diversity and endemism of plants (Mittermeier et al. 2011, Rezende et al. 2018). The Atlantic Forest covers about 15\% of the Brazilian territory, is distributed throughout the coast of the Brazilian coast from the State of Rio Grande do Sul to the Piauí, with forest patches in non-coastal states such as Goiás, Mato Grosso and Minas Gerais (SOS Mata Atlântica 2018). The Atlantic Forest has suffered from the effects of its fragmentation, the result of recurrent and disorderly deforestation that degrades and changes the distribution patterns of the species, causing incalculable and irreparable

1. Parte da Dissertação de Mestrado do primeiro Autor

2. Universidade Federal do Recôncavo da Bahia, Centro de Ciências Agrárias, Ambientais e Biológicas, Rua Rui Barbosa, 710, Centro, 44380-000 Cruz das Almas, BA, Brasil

3. Autor para correspondência: lidyanne.aona@gmail.com 
losses to the Brazilian biodiversity (Calgaro et al. 2015, Melo et al. 2015). This has reduced the number and size of plant and animal populations, increasing the loss of biodiversity and alleles that are fundamental for the adaptation and evolution of species (Calgaro et al. 2015, Melo et al. 2015). This reduction of the environment makes the Atlantic Forest fragile and compromises local biodiversity (Newbold et al. 2015).

The floristic knowledge is useful to record the species of plants occurring in a given location, what can be used to understand biogeographical patterns and help to restore degraded areas with local native species (Moro \& Martins 2011). This knowledge could be useful to characterize and compare it with other areas, as well as to provide useful data for biodiversity conservation of remnants of the Atlantic Forest is fundamental. Also, they contribute to understanding processes that involve the effects of the fragmentation, structure and diversity of species, which are essential to draw strategies of conservation of these environments (Mota et al. 2017). In addition, studies at local scales, whether surveying or taxonomic treatment, are fundamental to compose the knowledge of the flora and biogeography of the species, since most of the studies on a global scale have been treated as a compilation of studies at smaller scales (Thomas et al. 2012).

In the State of Bahia, most of the studies involving the Atlantic Forest have concentrated in the southern part of the State, a region that is comprised of several forest formations (ombrophilous and semideciduous forests, for example) and has much of the biome's floristic diversity (Mori et al. 1981, Sobrinho \& Queiroz 2005, Thomas et al. 2009). However, Carnaval \& Moritz (2008) have already addressed the need to extend studies to other tropical forest areas in northeastern Brazil that are relatively little known and highly impacted.

The Recôncavo da Bahia is a region where there is a shortage of floristic and ecological studies, except for a study carried out in Serra da Jiboia (Sobrinho \& Queiroz 2005), where there is little or almost nothing about its native forest remnants. There were recorded 116 species of Angiosperms for Serra da Copioba, of which 36 were from historical collections made between the 1950s and 1970s and deposited in the Alexandre Leal Costa Herbarium (ALCB), 15 species deposited in the Universidade Estadual de Feira de Santana Herbarium (HUEFS) and 70 species were deposited in the Recôncavo da Bahia Herbarium (HURB) some collects from the Serra da Copioba (Specieslink, 2016) and Virtual Reflora databases Herbarium (JBRJ 2012). Thus, the objective of this work was to increase the floristic studies in the region by surveying the Angiosperms occurring in the the Atlantic Rain Forest fragment in the Serra da Copioba, which has information on endemism, conservation and its ecological implications on local biodiversity.

\section{Materials and methods}

The study was carried out in Serra da Copioba, located in the city of São Felipe (Bahia State), Recôncavo da Bahia region (figure 1). The area has rugged relief and maximum elevation of 360 meters. Soil types belong to the interior and pre-littoral trays: yellow latosol, red-yellow alico latosol, dystrophic and podzolic. The climate varies from humid and dry submerged with an average annual temperature of $23.8^{\circ} \mathrm{C}$ and an average annual rainfall of 800 to $1100 \mathrm{~mm}$, with a higher rainfall incidence between November and January (SEI 2017).

The vegetation of Serra da Copioba can be characterized as a remnant of dense ombrophilous forest located on a coastal tableland with high rainfall and without the presence of a defined dry period. Due to anthropic actions the vegetation is in a secondary successional state, marked mainly by presence of light-demanding tree and the striking presence of exotic species

The survey was carried out through collections on the tracks to cover the largest possible territory, between October 2016 and October 2017, totaling ten excursions ranging from 1 to 4 days. The botanical material was processed following the technical standards recommended by Bridson \& Forman (1992). All the collected materials were deposited in the Recôncavo da Bahia Herbarium (HURB). The identifications of the specimens were made from a comparison with exsiccates previously identified in HURB and consultation with specialists and assistance of specialized bibliography.

The records of plants collected in Serra da Copioba deposited at the Universidade Estadual de Feira de Santana Herbarium (HUEFS) and the historical collections made between the years of 1950 and 1970 that are inserted in the Alexandre Leal Costa Herbarium (ALCB) were added to the floristic list. Data was accessed through the Centro de Referência em Informação Ambiental database (CRIA 2016). The data was verified, evaluated and the materials without possibility for identification or doubtful location were excluded.

The classification system adopted was APG IV (2016). To define the habit of the individuals, field observations were used, as well as descriptions on voucher labels (for herbarium research), following information in the literature (Gonçalves \& Lorenzi 2007). The species nomenclature and distribution were verified in the Flora do Brasil database (BFG 2015, Flora do Brasil 2020 under construction 2018). 


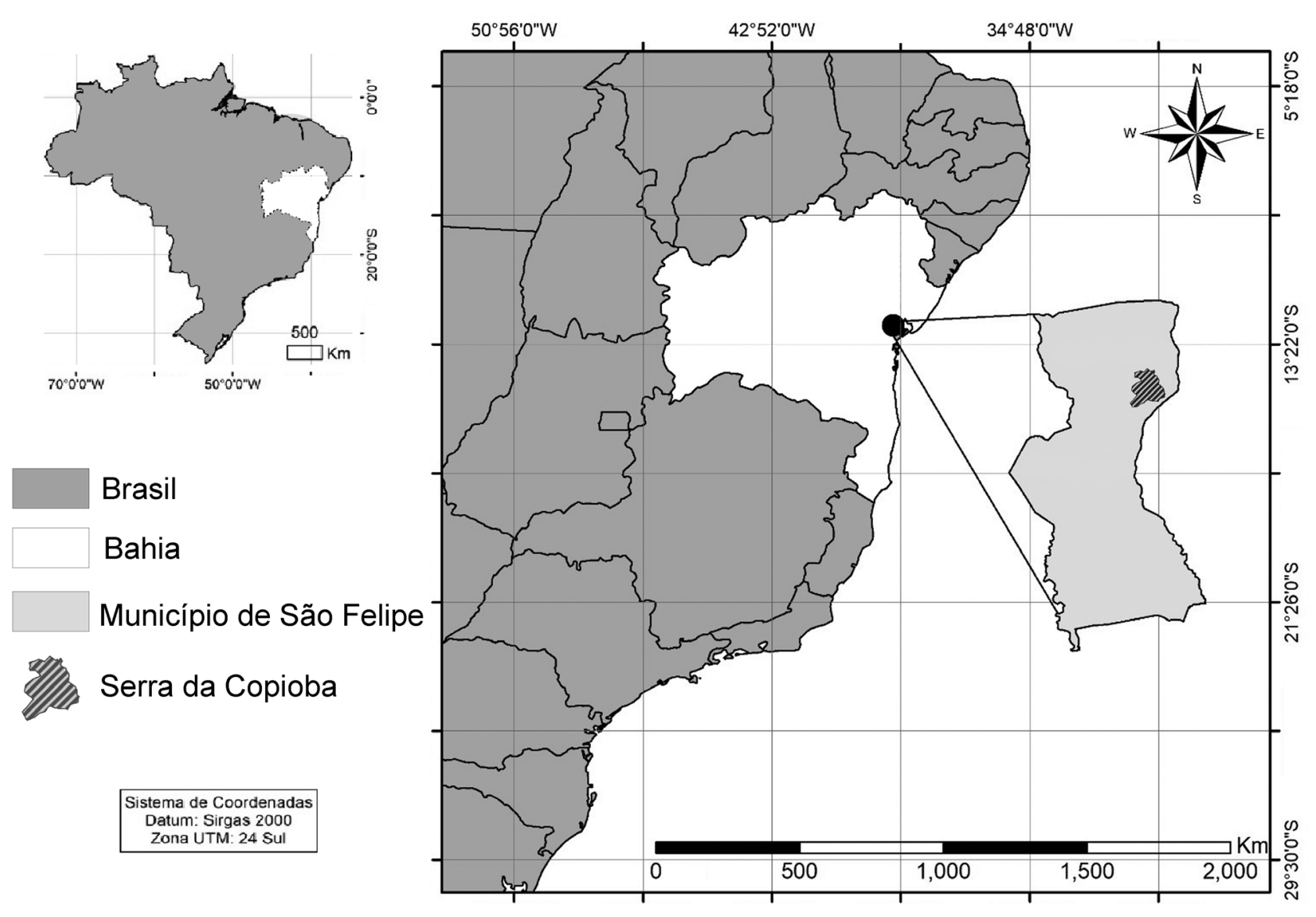

Figure 1: Location map of the study area, Serra da Copioba, São Felipe, Bahia State, Brazil.

\section{Results}

A total of 306 species of Angiosperms, distributed in 77 families and 200 genera were recorded for Serra da Copioba (table 1). In total, 273 species were identified up to the specific level $(88.9 \%), 19$ to genera $(6.5 \%)$ and 14 at the family level $(4.6 \%)$. The families with the highest number of species were: Rubiaceae (30 spp.); Fabaceae (19 spp.); Malvaceae (11 spp.), Asteraceae, Myrtaceae and Sapindaceae (10 spp.); Acanthaceae, Rutaceae (nine spp.); Araceae, Euphorbiaceae, Melastomataceae and Poaceae with (eight spp.); and Orchidaceae and Piperaceae (seven spp.). These 14 families comprise $50.3 \%$ of all species recorded in Serra da Copioba.

The endemism indexes recorded in this study are 23.2\% for endemic species of the Atlantic Forest (e.g. Aechmea depressa L.B.Sm. and Guatteria villosissima A.St.-Hil.) and $4.05 \%$ for endemic species of Bahia (e.g. Begonia delicata Gregório \& J.A.S. Costa, according to information of the List of Flora of Brazil (Flora do Brasil 2020, under construçtion). Three exotic species were recorded and identified: Artocarpus heterophyllus Lam., Cordyline australis Hook.f. and Citrus sp.

The historical collections made in the Serra da Copioba in the 1950s totaled 25 species (e.g. Asclepias curassavica L., Anthurium jilekii Schott and Abildgaardia ovata (Burm.f.) Kral) and 11 species in the 1970s (e.g., Gonzalagunia dicocca Cham. \& Schltdl., Erythroxylum nobile O.E.Schulz and Faramea hyacinthina Mart.). Although the sample effort of this study was much higher than those collected in the 50 s and 70 s, only seven species from the 25 collected in 1950 were collected.

The species Begonia reniformis Dryand (Begoniaceae), Cyperus luzulae (L.) (Cyperaceae), Erythroxylum citrifolium A.St.-Hil (Erythroxylaceae), Heliconia pendula Wawra (Heliconiaceae) and Begonia delicata Gregório \& J.A.S. Costa (Begoniaceae), are endemic to the Atlantic Forest. Of the 11 species collected in the 1970s, only five were collected: Gonzalagunia dicocca Cham. \& Schltdl. (Rubiaceae), Erythroxylum nobile O.E.Schulz (Erythroxylaceae), Faramea hyacinthina Mart. (Rubiaceae) and Margaritopsis chaenotricha (DC.) C.M.Taylor (Rubiaceae). 
Table 1. List of species occurring in Serra da Copioba, São Felipe, Bahia State, Brazil. shrub: shrub; tree: tree; epi: epiphyte; herb: herb; sub: subshrub; par: parasite; trep: vine. •: Collection of the 1950s; $\mathbf{m}$ : Collection of the 1970s; $\Delta$ : Retrieved in this work; $\boldsymbol{\Delta}$ : not collected in this work. AM: Amazonas; CAA: Caatinga; CER: Cerrado; PAN: Pantanal; MA: Mata Atlântica; PAM: Pampas.

\begin{tabular}{|c|c|c|c|}
\hline Family & Habit & Colector/Number & Phytogeographical Domain \\
\hline \multicolumn{4}{|l|}{ Acanthaceae } \\
\hline Aphelandra bahiensis (Nees) Wassh. & herb & Costa, G. 1051 & MA \\
\hline Aphelandra nitida Nees \& Mart. & sub & Moreira, D.M. 55 & MA \\
\hline Justicia antirrhina Nees \& Mart. & herb & Moreira, D.M. 52 & MA \\
\hline $\begin{array}{l}\text { Lepidagathis nemoralis (Mart. ex Nees) } \\
\text { Kameyama } \bullet\end{array}$ & sub & Lordêlo, R.P. 56-572 & MA \\
\hline Ruellia affinis (Schrad.) Lindau & sub & Moreira, D.M. 79 & MA \\
\hline Ruellia bahiensis (Nees) Morong & sub & Conceição, S. F. 807 & CAA \\
\hline Ruellia incompta (Ness) Lindau & sub & Moreira, D.M. 81 & AM, CAA, CER, PAN, MA \\
\hline Ruellia paniculata $\mathrm{L}$. & shrub & Moreira, D.M. 60 & CAA, CER, MA \\
\hline Schaueria gonatistachya Nees & sub & Moreira, D.M. 54 & MA \\
\hline \multicolumn{4}{|l|}{ Alstroemeriaceae } \\
\hline Bomarea edulis (Tussac) Herb. $\bullet \mathbf{} \Delta$ & vine & Moreira, D.M. 227 & AM, CAA, CER, PAN, MA \\
\hline \multicolumn{4}{|l|}{ Amaranthaceae } \\
\hline $\begin{array}{l}\text { Alternanthera brasiliana var. brasiliana (L.) } \\
\text { Kuntz }\end{array}$ & shrub & Moreira, D.M. 163 & AM, CAA, CER, MA \\
\hline $\begin{array}{l}\text { Alternanthera brasiliana var. vilosa (Moq.) } \\
\text { Kuntz }\end{array}$ & shrub & Conceição, S.F. 815 & AM, CAA, CER, MA \\
\hline \multicolumn{4}{|l|}{ Anacardiaceae } \\
\hline Astronium concinnum Schott & tree & $\begin{array}{l}\text { Souza, J.S. } \\
\text { (HURB 20475) }\end{array}$ & CAA, CER, MA \\
\hline Schinus terebinthifolius Raddi & tree & Moreira, D.M. 119 & AM, CAA, CER, PAN, MA \\
\hline Spondias venulosa (Engl.) Engl. & tree & $\begin{array}{c}\text { Souza, J.S. } \\
\text { (HURB 20492) }\end{array}$ & MA \\
\hline \multicolumn{4}{|l|}{ Annonaceae } \\
\hline Annona cacans Warm. & tree & $\begin{array}{l}\text { Souza, J.S. } \\
\text { (HURB 20482) }\end{array}$ & MA \\
\hline Guatteria villosissima A.St.-Hil. & tree & $\begin{array}{c}\text { Souza, J.S. } \\
\text { (HURB 20438) }\end{array}$ & MA \\
\hline Guatteria sp. & shrub & Moreira, D.M.50 & $*$ \\
\hline Unonopsis guatterioides (A.DC.) R.E.Fr. & tree & $\begin{array}{c}\text { Souza, J.S. } \\
\text { (HURB 20461) }\end{array}$ & $\mathrm{AM}, \mathrm{CE}, \mathrm{MA}$ \\
\hline Xylopia sericia A.St.-Hil. & tree & Moreira, D.M. 104 & AM, CER, MA \\
\hline \multicolumn{4}{|l|}{ Apiaceae } \\
\hline Spananthe paniculata Jacq. & sub & Costa, G. 1536 & CAA, CER, MA \\
\hline \multicolumn{4}{|l|}{ Apocynaceae } \\
\hline 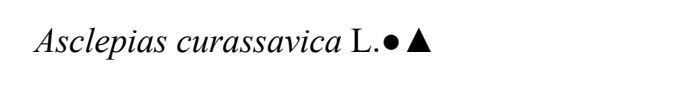 & sub & Lordêlo, R.P. 56-617 & $\begin{array}{c}\text { AM, CAA, CER, PAN, PAM, } \\
\text { MA }\end{array}$ \\
\hline Himatanthus obovatus (Müll. Arg.) Woodson & tree & Moreira, D.M. 36 & AM, CAA, CER \\
\hline $\begin{array}{l}\text { Mandevilla scabra (Hoffmanns. ex Roem. \& } \\
\text { Schult.) K.Schum. }\end{array}$ & vine & Conceição, S.F. 808 & AM, CAA, CER, MA \\
\hline
\end{tabular}


Table 1 (continuation)

\begin{tabular}{|c|c|c|c|}
\hline Family & Habit & Colector/Number & Phytogeographical Domain \\
\hline Tabernaemontana hystrix Steud. & tree & $\begin{array}{c}\text { Souza, J.S. } \\
\text { (HURB 20503) }\end{array}$ & CER, MA \\
\hline \multicolumn{4}{|l|}{ Araceae } \\
\hline Anthurium affine Schott & herb & Moreira, D.M. 260 & CAA, CER, MA \\
\hline Anthurium bellum Schott & herb & Moreira, D.M. 74 & MA \\
\hline Anthurium jilekii Schott $\bullet \mathbf{\Delta}$ & herb & Pinto, G.C.P. 1050 & MA \\
\hline Anthurium pentaphyllum (Aubl.) G.Don & herb & Andrade, I.M. 810 & AM, MA, PAN \\
\hline Anthurium radicans K.Koch \& Haage & sub & Moreira, D.M. 202 & MA \\
\hline Heteropsis oblongifolia Kunth & herb & Andrade, I.M. 828 & AM, CER, MA \\
\hline Monstera adansonii Schott & herb & Andrade, I.M. 2771 & AM, CAA, CER, MA \\
\hline Philodendron pedatum (Hook.) Kunth & herb & Andrade, I.M. 2744 & AM, CAA, CER, MA \\
\hline \multicolumn{4}{|l|}{ Arecaceae } \\
\hline Attalea funifera Mart. & palm & Costa, G. 1565 & MA \\
\hline \multicolumn{4}{|l|}{ Aristolochiaceae } \\
\hline Aristolochia labiata Willd. & vine & Costa, G. 1055 & CAA, CER, MA \\
\hline \multicolumn{4}{|l|}{ Asteraceae } \\
\hline Achyrocline flaccida (Weinm.) DC. & herb & Costa, G. 1553 & CER, MA, PAM, PAN \\
\hline Achyrocline saturejoides (Lam.) DC. & shrub & Moreira, D.M. 7 & CER, MA, PAM \\
\hline Baccharis cinerea DC. & shrub & Moreira, D.M. 127 & CAA, CER, MA \\
\hline Blanchetia heterotricha DC. & shrub & Costa, G. 1545 & CAA, MA \\
\hline $\begin{array}{l}\text { Chromolaena odorata (L.) R.M.King \& } \\
\text { H.Rob. }\end{array}$ & shrub & Costa, G. 1542 & $\begin{array}{c}\text { AM, CAA, CER, PAN, PAM, } \\
\text { MA }\end{array}$ \\
\hline Cyrtocymura mattos-silvae (H.Rob.) H.Rob. & shrub & Moreira, D.M. 11 & MA \\
\hline $\begin{array}{l}\text { Lepidaploa cotoneaster (Willd. ex Spreng.) } \\
\text { H.Rob. }\end{array}$ & shrub & Moreira, D.M. 6 & CER \\
\hline Rolandra fruticosa (L.) Kuntze & shrub & Costa, G. 1053 & $\mathrm{AM}, \mathrm{CAA}$ \\
\hline Verbesina macrophylla (Cass.) S.F.Blake & shrub & Moreira, D.M. 232 & CAA, MA \\
\hline Vernonanthura brasiliana (L.) H.Rob. & shrub & Moreira, D.M. 13 & AM, CAA, CER \\
\hline \multicolumn{4}{|l|}{ Begoniaceae } \\
\hline $\begin{array}{l}\text { Begonia delicata Gregório \& J.A.S. Costa } \\
\bullet \Delta\end{array}$ & shrub & Moreira, D.M. 10 & MA \\
\hline Begonia reniformis Dryand. $\bullet \Delta$ & herb & Moreira, D.M. 192 & CAA, CER, MA \\
\hline \multicolumn{4}{|l|}{ Bignoniaceae } \\
\hline Adenocalymma marginatum (Cham.) DC. & vine & Moreira, D.M. 77 & MA, PAM \\
\hline \multicolumn{4}{|l|}{ Bignoniaceae } \\
\hline Adenocalymma coriaceum A.DC. & vine & Moreira, D.M. 149 & MA \\
\hline $\begin{array}{l}\text { Fridericia bahiensis (Schauer ex. DC.) } \\
\text { L.G.Lohmann }\end{array}$ & tree & Moreira, D.M. 234 & CAA, CER, MA \\
\hline $\begin{array}{l}\text { Handroanthus chrysotrichus (Mart. ex DC.) } \\
\text { Mattos }\end{array}$ & tree & $\begin{array}{c}\text { Souza, J.S. } \\
\text { (HURB 20445) }\end{array}$ & $\begin{array}{c}\text { AM, CAA, CER, PAN, PAM, } \\
\text { MA }\end{array}$ \\
\hline $\begin{array}{l}\text { Handroanthus impetiginosus (Mart. ex DC.) } \\
\text { Mattos }\end{array}$ & tree & $\begin{array}{c}\text { Souza, J.S. } \\
\text { (HURB.20446) }\end{array}$ & $\begin{array}{c}\text { AM, CAA, CER, PAN, PAM, } \\
\text { MA }\end{array}$ \\
\hline
\end{tabular}


Table 1 (continuation)

\begin{tabular}{lccc}
\hline Family & Habit & Colector/Number & Phytogeographical Domain \\
\hline Tabebuia roseoalba (Ridl.) Sandwith & tree & $\begin{array}{c}\text { Souza, J.S. } \\
\text { (HURB 20435) }\end{array}$ & CAA, CER, MA
\end{tabular}

Boraginaceae

Cordia corymbosa (Desv.) Don

Cordia lomatoloba I.M.Johnst.

Cordia superba Cham.

Cordia sp.

Myriopus villosus (Salzm. ex DC.)

J.I.M.Melo

Varronia curassavica Jacq.

Bromeliaceae

Aechmea depressa L.B.Sm.

Aechmea fulgens Brongn.

Billbergia saundersii Bull

Canistrum sp.

Hohenbergia stellata Schult. \& Schult.f.

sp. 1

Burseraceae

Protium spruceanum (Benth.) Engl.

Cactaceae

Brasiliopuntia brasiliensis (Willd.) A.Berger

Hylocereus setaceus (Salm-Dyck) R.Bauer

Rhipsalis oblonga Loefgr.

Cannabaceae

Celtis pubescens (Kunth) Spreng.

Celtis spinosa Spreng.

Capparaceae

Cynophalla flexuosa (L.) J.Presl

Crateva tapia $\mathrm{L}$.

Monilicarpa brasiliana (Banks ex DC.)

Cornejo \& Iltis.

Neocalyptrocalyx grandipetala (Maguire \&

Steyerm.) Cornejo \& Iltis

Celastraceae

Monteverdia ilicifolia (Mart. ex Reissek) Biral

Monteverdia sp.

Clusiaceae

\begin{tabular}{|c|c|c|}
\hline$*$ & Moreira, D.M. 72 & $\begin{array}{c}\text { AM, CAA, CER, PAN, PAM, } \\
\text { MA }\end{array}$ \\
\hline tree & Lordêlo, R.P. 56675 & $\mathrm{AM}$ \\
\hline tree & Moreira, D.M. 120 & CAA, CER, MA \\
\hline shrub & Moreira, D.M. 247 & $*$ \\
\hline$*$ & Moreira, D.M. 19 & CAA, MA \\
\hline sub & Moreira, D.M. 125 & AM, CAA, CER, MA \\
\hline terr & Moreira, D.M. 144 & MA \\
\hline terr & Moreira, D.M. 170 & MA \\
\hline terr & Moreira, D.M. 150 & MA \\
\hline terr & Moreira, D.M. 169 & $*$ \\
\hline terr & Moreira, D.M. 171 & MA, CAA \\
\hline terr & Moreira, D.M. 168 & $*$ \\
\hline tree & $\begin{array}{c}\text { Souza, J.S. } \\
\text { (HURB 20494) }\end{array}$ & AM, CER, MA \\
\hline tree & Moreira, D.M. 221 & AM, CAA, CER, MA \\
\hline herb & Moreira, D.M. 261 & AM, CAA, CER, MA \\
\hline epi & Moreira, D.M. 108 & AM, CAA, CER, MA \\
\hline shrub & Moreira, D.M. 30 & $\begin{array}{c}\text { AM, CAA, CER, PAN, PAM, } \\
\text { MA }\end{array}$ \\
\hline shrub & Moreira, D.M. 135 & $\begin{array}{c}\text { AM, CAA, CER, PAN, PAM, } \\
\text { MA }\end{array}$ \\
\hline
\end{tabular}

tree Souza, J.S. (HURB 20479)

tree $\quad$ Moreira, D.M. 24

Souza, J.S.

(HURB 20447)

tree $\quad$ Moreira, D.M. 267

Souza, J.S. (HURB 20418)

* $\quad$ Moreira, D.M. 148

AM, CER, CAA, MA, PAN

AM, CAA, CER, MA

MA, PAN

MA, AM

CE, MA, PAM 
Table 1 (continuation)

\begin{tabular}{|c|c|c|c|}
\hline Family & Habit & Colector/Number & Phytogeographical Domain \\
\hline Garcinia brasiliensis Mart. & tree & $\begin{array}{c}\text { Souza, J.S. } \\
\text { (HURB 20434) }\end{array}$ & MA \\
\hline \multicolumn{4}{|l|}{ Commelinaceae } \\
\hline Commelina erecta $\mathrm{L}$. & herb & Moreira, D.M. 87 & AM, CAA, CER, PAN, MA \\
\hline Dichorisandra hexandra (Aubl.) C.B.Clarke & shrub & Moreira, D.M. 181 & AM, CAA, CER, MA \\
\hline Dichorisandra sp. & herb & Moreira, D.M. 263 & $*$ \\
\hline \multicolumn{4}{|l|}{ Connaraceae } \\
\hline Connarus regnellii $\mathrm{G}$. Schellenb. & tree & $\begin{array}{c}\text { Souza, J.S. } \\
\text { (HURB 20491) }\end{array}$ & MA \\
\hline \multicolumn{4}{|l|}{ Convolvulaceae } \\
\hline Ipomoea sp. & vine & Moreira, D.M. 18 & $*$ \\
\hline \multicolumn{4}{|l|}{ Costaceae } \\
\hline Costus scaber Ruiz \& Pav. & sub & Moreira, D.M. 64 & AM, CER, MA \\
\hline \multicolumn{4}{|l|}{ Cucurbitaceae } \\
\hline sp. 1 & herb & Moreira, D.M. 266 & $*$ \\
\hline \multicolumn{4}{|l|}{ Cyperaceae } \\
\hline Abildgaardia ovata (Burm.f.) Kral $\bullet \mathbf{\Delta}$ & herb & Lordêlo, R.P. 56-396 & AM, CAA, CER, MA \\
\hline Andropogon bicornis L. & herb & Costa, G. 1562 & $\begin{array}{c}\text { AM, CAA, CER, PAN, MA, } \\
\text { PAM }\end{array}$ \\
\hline Cyperus luzulae (L.) Retz. $\bullet \Delta$ & herb & Moreira, D.M. 131 & $\begin{array}{c}\text { AM, CAA, CER, PAN, MA, } \\
\text { PAM }\end{array}$ \\
\hline Fuirena umbellata Rottb. & herb & Moreira, D.M. 235 & $\begin{array}{c}\text { AM, CAA, CER, PAN, MA, } \\
\text { PAM }\end{array}$ \\
\hline \multicolumn{4}{|l|}{ Dioscoreaceae } \\
\hline Dioscorea altissima Lam. & vine & Moreira, D.M. 246 & AM, CAA, CER, MA \\
\hline \multicolumn{4}{|l|}{ Ebenaceae } \\
\hline Diospyros inconstans Jacq. & shrub & Moreira, D.M. 213 & AM, CAA, CER, PAN, MA \\
\hline \multicolumn{4}{|l|}{ Erythroxylaceae } \\
\hline Erythroxylum citrifolium A.St.-Hil. $\bullet \Delta$ & tree & Moreira, D.M. 107 & AM, CER, MA \\
\hline Erythroxylum nobile O.E.Schulz $\mathbf{\square \Delta}$ & tree & Moreira, D.M. 101 & MA \\
\hline Erythroxylum pelleterianum A.St.-Hil. & tree & $\begin{array}{c}\text { Souza, J.S. } \\
\text { (HURB 20472) }\end{array}$ & AM, CER, MA \\
\hline \multicolumn{4}{|l|}{ Euphorbiaceae } \\
\hline Acalypha brasiliensis Müll.Arg. & sub & Moreira, D.M. 159 & AM, CAA, CER, MA \\
\hline Acalypha multicaulis Müll.Arg. & sub & Conceição, S.F. 815 & CAA, CER, MA, PAM \\
\hline Actinostemon concolor (Spreng.) Müll.Arg. & tree & $\begin{array}{c}\text { Souza, J.S. } \\
\text { (HURB 20518) }\end{array}$ & AM, CAA, MA \\
\hline Actinostemon klotzschii (Didr.) Pax & tree & $\begin{array}{c}\text { Souza, J.S. } \\
\text { (HURB 20517) }\end{array}$ & AM, MA \\
\hline Actinostemon verticillatus (Klotzsch) Baill. & tree & $\begin{array}{c}\text { Souza, J.S. } \\
\text { (HURB 20487) }\end{array}$ & MA \\
\hline Croton heliotropiifolius Kunth & sub & Costa, G. 1561 & AM, CAA, CER, MA \\
\hline
\end{tabular}


Table 1 (continuation)

\begin{tabular}{|c|c|c|c|}
\hline Family & Habit & Colector/Number & Phytogeographical Domain \\
\hline Mabea piriri Aubl. & tree & $\begin{array}{c}\text { Souza, J.S. } \\
\text { (HURB 20450) }\end{array}$ & AM, CER, MA \\
\hline Senefeldera verticillata (Vell.) Croizat & tree & $\begin{array}{c}\text { Souza, J.S. } \\
\text { (HURB 20509) }\end{array}$ & MA \\
\hline \multicolumn{4}{|l|}{ Fabaceae } \\
\hline $\begin{array}{l}\text { Albizia polycephala (Benth.) Killip ex } \\
\text { Record }\end{array}$ & tree & $\begin{array}{l}\text { Souza, J.S. } \\
\text { (HURB 20443) }\end{array}$ & CAA, CER, MA \\
\hline Andira fraxinifolia Benth. & tree & Conceição, S.F. 799 & CAA, CER, MA \\
\hline Diplotropis ferruginea Benth. & tree & $\begin{array}{c}\text { Souza, J.S. } \\
\text { (HURB 20440) }\end{array}$ & MA \\
\hline Hymenaea courbaril L. & tree & $\begin{array}{c}\text { Souza, J.S. } \\
\text { (HURB 20455) }\end{array}$ & AM, CAA, CER, PAN, MA \\
\hline Inga capitata Desv. & tree & $\begin{array}{c}\text { Souza, J.S. } \\
\text { (HURB 20466) }\end{array}$ & AM, MA \\
\hline Inga edulis Mart. & tree & Moreira, D.M. 136 & AM, CAA, CER, MA \\
\hline Inga ciliata C.Presl & shrub & Moreira, D.M. 242 & AM, MA \\
\hline Inga striata Benth. & tree & $\begin{array}{l}\text { Souza, J.S. } \\
\text { (HURB 20464) }\end{array}$ & AM, CER, CAA \\
\hline Macroptilium sp. & vine & Moreira, D.M. 139 & * \\
\hline Myroxylon peruiferum L.f. & tree & $\begin{array}{c}\text { Souza, J.S. } \\
\text { (HURB 20439) }\end{array}$ & $\mathrm{CE}, \mathrm{MA}$ \\
\hline Ormosia fastigiata Tul. & tree & $\begin{array}{c}\text { Souza, J.S. } \\
\text { (HURB 20441) }\end{array}$ & $\mathrm{CE}, \mathrm{MA}$ \\
\hline $\begin{array}{l}\text { Pseudopiptadenia brenanii G.P.Lewis \& } \\
\text { M.P.Lima }\end{array}$ & tree & $\begin{array}{c}\text { Souza, J.S. } \\
\text { (HURB 20444) }\end{array}$ & CAA, CER \\
\hline Senegalia polyphylla (DC.) Britton \& Rose & tree & $\begin{array}{l}\text { Souza, J.S. } \\
\text { (HURB 20442) }\end{array}$ & AM, CAA, CER, PAN, MA \\
\hline Senna affinis (Benth.) H.S.Irwin \& Barneby & $*$ & Moreira, D.M. 132 & $\mathrm{CE}, \mathrm{MA}$ \\
\hline $\begin{array}{l}\text { Senna macranthera (DC. Ex Collad.) } \\
\text { H.S.Irwin \& Barneby }\end{array}$ & tree & Moreira, D.M. 231 & CAA, CER, MA \\
\hline Swartzia simplex (Sw.) Spreng. & tree & $\begin{array}{c}\text { Souza, J.S. } \\
\text { (HURB 20445) }\end{array}$ & MA \\
\hline Zollernia cowanii Mansano & tree & Moreira, D.M. 51 & MA \\
\hline sp. 1 & vine & Moreira, D.M. 100 & * \\
\hline sp. 2 & vine & Moreira, D.M. 138 & * \\
\hline \multicolumn{4}{|l|}{ Gentianaceae } \\
\hline Coutoubea spicata Aubl. & $*$ & Costa, G. 1539 & AM, CER, MA \\
\hline Schultesia gracilis Mart. & herb & Conceição, S.F. 810 & CE, MA \\
\hline \multicolumn{4}{|l|}{ Gesneriaceae } \\
\hline $\begin{array}{l}\text { Sinningia barbata (Nees \& Mart.) } \\
\text { G.Nicholson }\end{array}$ & sub & Moreira, D.M. 59 & MA \\
\hline \multicolumn{4}{|l|}{ Heliconiaceae } \\
\hline Heliconia pendula Wawra $\bullet \Delta$ & sub & Moreira, D.M. 73 & MA \\
\hline Heliconia psittacorum L.f. & herb & Conceição, S.F. 817 & AM, CA, CER, PAN, MA \\
\hline
\end{tabular}


Table 1 (continuation)

\begin{tabular}{|c|c|c|c|}
\hline Family & Habit & Colector/Number & Phytogeographical Domain \\
\hline \multicolumn{4}{|l|}{ Hydroleaceae } \\
\hline Hydrolea spinosa $\mathrm{L}$. & sub & Conceição, S.F. 809 & $\begin{array}{l}\text { AM, CA, CER, PAN, MA, } \\
\text { PAM }\end{array}$ \\
\hline \multicolumn{4}{|l|}{ Hypericaceae } \\
\hline Vismia guianensis (Aubl.) Pers. & tree & Moreira, D.M. 106 & AM, CAA, CER, MA \\
\hline \multicolumn{4}{|l|}{ Iridaceae } \\
\hline Cipura paludosa Aubl. & herb & Moreira, D.M. 204 & AM, CAA, CER, MA \\
\hline \multicolumn{4}{|l|}{ Lamiaceae } \\
\hline $\begin{array}{l}\text { Mesosphaerum irwinii (Harley) Harley \& } \\
\text { J.F.B.Pastore }\end{array}$ & sub & Conceição, S.F. 800 & CAA \\
\hline sp. 1 & herb & Conceição, S.F. 811 & $*$ \\
\hline \multicolumn{4}{|l|}{ Lauraceae } \\
\hline Aniba firmula (Nees \& Mart.) Mez & tree & $\begin{array}{l}\text { Souza, J.S. } \\
\text { (HURB 20514) }\end{array}$ & AM, CER, MA \\
\hline Endlicheria paniculata (Spreng.) J.F.Macbr. & tree & $\begin{array}{c}\text { Souza, J.S. } \\
\text { (HURB 20504) }\end{array}$ & AM, CAA, CER, MA, PAN \\
\hline Ocotea brachybotrya (Meisn.) Mez & tree & $\begin{array}{c}\text { Souza, J.S. } \\
\text { (HURB 20456) }\end{array}$ & MA \\
\hline Ocotea indecora (Schott) Mez & tree & $\begin{array}{c}\text { Souza, J.S. } \\
\text { (HURB 20457) }\end{array}$ & MA \\
\hline Ocotea laxa (Nees) Mez & tree & $\begin{array}{l}\text { Souza, J.S. } \\
\text { (HURB 20507) }\end{array}$ & MA \\
\hline Ocotea velutina (Nees) Rohwer $\bullet \boldsymbol{\Delta}$ & tree & Lordêlo, R.P. 5637 & MA, CER \\
\hline \multicolumn{4}{|l|}{ Laxmanniaceae } \\
\hline Cordyline australis Hook.f. & tree & Moreira, D.M. 34 & EXOTIC \\
\hline \multicolumn{4}{|l|}{ Lecythidaceae } \\
\hline $\begin{array}{l}\text { Eschweilera ovata (Cambess.) Mart. ex } \\
\text { Miers }\end{array}$ & tree & $\begin{array}{l}\text { Souza, J.S. } \\
\text { (HURB 20497) }\end{array}$ & AM, MA \\
\hline \multicolumn{4}{|l|}{ Linderniaceae } \\
\hline $\begin{array}{l}\text { Cubitanthus alatus (Cham. \& Schltdl.) } \\
\text { Barringer } \bullet \boldsymbol{\Lambda}\end{array}$ & herb & Lordêlo, R.P. 56-329 & MA \\
\hline \multicolumn{4}{|l|}{ Loasaceae } \\
\hline Aosa parviflora (Schrad. ex DC.) Weigend & vine & Moreira, D.M. 203 & MA \\
\hline \multicolumn{4}{|l|}{ Loranthaceae } \\
\hline Phthirusa sp. & tree & Moreira, D.M. 137 & $*$ \\
\hline \multicolumn{4}{|l|}{ Lythraceae } \\
\hline Cuphea brachypoda T.B.Cavalc. & sub & Conceição, S.F. 797 & CER \\
\hline Cuphea racemosa (L.f.) Spreng. & herb & Costa, G. 1532 & $\begin{array}{l}\text { AM, CA, CER, PAN, MA, } \\
\text { PAM }\end{array}$ \\
\hline \multicolumn{4}{|l|}{ Malpighiaceae } \\
\hline Byrsonima sericea DC. & shrub & Moreira, D.M. 122 & AM, CAA, CER, MA \\
\hline Heteropterys sanctorum W.R.Anderson $\bullet \boldsymbol{\Delta}$ & vine & Lordêlo, R.P. 56 & MA \\
\hline Tetrapterys sp. & sub & Moreira, D.M. 57 & $*$ \\
\hline
\end{tabular}


Table 1 (continuation)

\begin{tabular}{|c|c|c|c|}
\hline Family & Habit & Colector/Number & Phytogeographical Domain \\
\hline \multicolumn{4}{|l|}{ Malvaceae } \\
\hline Ceiba speciosa (A.St.-Hil.) Ravenna & tree & $\begin{array}{l}\text { Souza, J.S. } \\
\text { (HURB 20480) }\end{array}$ & AM, CAA, CER, MA \\
\hline $\begin{array}{l}\text { Eriotheca pentaphylla (Vell. \& K.Schum.) } \\
\text { A.Roby** }\end{array}$ & tree & $\begin{array}{c}\text { Souza, J.S. } \\
\text { (HURB 20463) }\end{array}$ & MA \\
\hline Helicteres corylifolia Nees \& Mart & $*$ & Costa, G. 1057 & AM, CAA, CER, MA \\
\hline $\begin{array}{l}\text { Pavonia fruticosa (Mill.) Fawc. \& Rendle } \\
\text { - } \Delta\end{array}$ & shrub & Lordêlo, R.P. 56-331 & AM, MA \\
\hline Pavonia malacophylla (Link \& Otto) Garcke & shrub & Moreira, D.M. 99 & AM, CAA, CER, MA \\
\hline Pavonia martii Colla & $*$ & Moreira, D.M. 134 & CAA, CER \\
\hline Sida cordifolia L. & shrub & Moreira, D.M. 140 & AM, CAA, CER, MA \\
\hline Sida glomerata Cav. & $* *$ & Costa, G. 39 & AM, CAA, CER, MA, PAN \\
\hline Sida indica $\mathrm{L}$. & sub & Moreira, D.M. 23 & $*$ \\
\hline Sidastrum paniculatum (L.) Fryxell & $*$ & Costa, G. 1544 & AM, CAA, CER, MA \\
\hline Triumfetta semitriloba Jacq. & $*$ & Costa, G. 155 & AM, CAA, CER, MA \\
\hline \multicolumn{4}{|l|}{ Marantaceae } \\
\hline $\begin{array}{l}\text { Goeppertia oblonga (Mart.) Borchs \& S. } \\
\text { Suárez } \bullet \Delta\end{array}$ & herb & Moreira, D.M. 225 & MA \\
\hline $\begin{array}{l}\text { Goeppertia cylindrica (Roscoe) Borchs. \& } \\
\text { S.Suárez }\end{array}$ & herb & Moreira, D.M. 206 & CER, MA \\
\hline Stromanthe porteana Griseb. & herb & Moreira, D.M. 103 & CER, MA \\
\hline \multicolumn{4}{|l|}{ Melastomataceae } \\
\hline Clidemia hirta (L.) D.Don & sub & Conceição, S.F. 796 & AM, CAA, CER, MA \\
\hline Miconia francavillana Cogn. & shrub & Moreira, D.M. 42 & MA \\
\hline Miconia minutiflora (Bonpl.) DC. & $*$ & Costa, G. 1547 & $\mathrm{AM}, \mathrm{CAA}, \mathrm{CER}, \mathrm{MA}$ \\
\hline Miconia nervosa (Sm.) Triana $\bullet \mathbf{\Delta}$ & shrub & Lordêlo, R.P. 5730 & $\mathrm{AM}, \mathrm{CAA}, \mathrm{CER}, \mathrm{MA}$ \\
\hline Miconia prasina (Sw.) DC. & tree & Moreira, D.M. 123 & $\mathrm{AM}, \mathrm{CAA}, \mathrm{CER}, \mathrm{MA}$ \\
\hline Miconia sp. 1 & arb & Moreira, D.M. 63 & $*$ \\
\hline Pleroma clidemioides Berg. ex Trianna & shrub & Moreira, D.M. 94 & MA \\
\hline sp. 1 & tree & Moreira, D.M. 185 & $*$ \\
\hline \multicolumn{4}{|l|}{ Meliaceae } \\
\hline Guarea guidonia (L.) Sleumer & tree & Moreira, D.M. 141 & AM, CAA, CER, MA \\
\hline Guarea macrophylla Vahl & tree & Moreira, D.M. 254 & AM, CAA, CER, MA \\
\hline Trichilia hirta L. & shrub & Moreira, D.M. 29 & AM, CAA, CER, MA \\
\hline Trichilia lepidota Mart. & tree & Moreira, D.M. 248 & MA \\
\hline Trichilia pallens C.DC. & tree & $\begin{array}{l}\text { Souza, J.S. (HURB } \\
\text { 20465) }\end{array}$ & $\mathrm{CE}, \mathrm{MA}$ \\
\hline Trichilia pallida Sw. & tree & $\begin{array}{l}\text { Souza, J.S. (HURB } \\
\text { 20483) }\end{array}$ & $\mathrm{AM}, \mathrm{CER}, \mathrm{MA}$ \\
\hline \multicolumn{4}{|l|}{ Menispermaceae } \\
\hline $\begin{array}{l}\text { Chondrodendron microphyllum (Eichler) } \\
\text { Moldenke }\end{array}$ & tree & Moreira, D.M. 75 & MA \\
\hline
\end{tabular}


Table 1 (continuation)

\begin{tabular}{|c|c|c|c|}
\hline Family & Habit & Colector/Number & Phytogeographical Domain \\
\hline $\begin{array}{l}\text { Chondrodendron platiphyllum (A.St.-Hil.) } \\
\text { Miers }\end{array}$ & vine & Moreira, D.M.265 & MA \\
\hline Cissampelos andromorpha DC. & vine & Moreira, D.M. 112 & $\begin{array}{l}\text { AM, CAA, CER, MAT, PAN, } \\
\text { PAM }\end{array}$ \\
\hline \multicolumn{4}{|l|}{ Monimiaceae } \\
\hline Mollinedia elegans Tul. & $*$ & Moreira, D.M. 20901 & MA \\
\hline Mollinedia triflora (Spreng.) Tul. & shrub & Moreira, D.M. 56 & MA \\
\hline \multicolumn{4}{|l|}{ Moraceae } \\
\hline Artocarpus heterophyllus Lam. & tree & Moreira, D.M. 274 & EXOTIC \\
\hline Ficus adhatodifolia Schott in Spreng. & tree & $\begin{array}{c}\text { Souza, J.S. } \\
\text { (HURB 20454) }\end{array}$ & CAA, CER, MA, PAN \\
\hline Sorocea bonplandii (Baill.) W.C.Burger et al. & tree & $\begin{array}{c}\text { Souza, J.S. } \\
\text { (HURB 20481) }\end{array}$ & CER, MA \\
\hline Sorocea hilarii Gaudich. & shrub & Moreira, D.M. 155 & CER, MA \\
\hline \multicolumn{4}{|l|}{ Myrtaceae } \\
\hline Calyptranthes widgreniana O.Berg & tree & $\begin{array}{c}\text { Souza, J.S. } \\
\text { (HURB 20513) }\end{array}$ & CER, MA \\
\hline Eugenia excoriata O.Berg & tree & $\begin{array}{c}\text { Souza, J.S. } \\
\text { (HURB 20497) }\end{array}$ & MA \\
\hline Eugenia florida DC. & tree & $\begin{array}{c}\text { Souza, J.S. } \\
\text { (HURB 20432) }\end{array}$ & $\mathrm{AM}, \mathrm{CAA}, \mathrm{CER}, \mathrm{MA}$ \\
\hline Eugenia expansa Spring ex Mart. & shrub & Moreira, D.M. 146 & MA \\
\hline Myrcia splendens (Sw.) DC. • $\mathbf{\Delta}$ & tree & Lordêlo, R.P. 352 & AM, CAA, CER, MA, PAN \\
\hline Myrcia venulosa DC. & tree & $\begin{array}{l}\text { Souza, J.S. } \\
\text { (HURB 20477) }\end{array}$ & CER, MA \\
\hline Psidium cauliflorum Landrum \& Sobral & tree & $\begin{array}{c}\text { Souza, J.S. } \\
\text { (HURB 20471) }\end{array}$ & MA \\
\hline Psidium guineense SW. & tree & Moreira, D.M. 113 & AM, CAA, CER, MA \\
\hline Psidium sp. 1 & tree & Moreira, D.M. 130 & $*$ \\
\hline sp. 1 & shrub & Moreira, D.M. 216 & $*$ \\
\hline \multicolumn{4}{|l|}{ Nyctaginaceae } \\
\hline Guapira opposita (Vell.) Reitz & tree & $\begin{array}{c}\text { Souza, J.S. } \\
\text { (HURB 20490) }\end{array}$ & $\mathrm{AM}, \mathrm{CAA}, \mathrm{CER}, \mathrm{MA}$ \\
\hline Guapira venosa (Choisy) Lundell & tree & $\begin{array}{c}\text { Souza, J.S. } \\
\text { (HURB 20510) }\end{array}$ & AM, MA \\
\hline Pisonia zapallo Griseb. & tree & $\begin{array}{c}\text { Souza, J.S. } \\
\text { (HURB 20516) }\end{array}$ & MA, PAN \\
\hline \multicolumn{4}{|l|}{ Olacaceae } \\
\hline Heisteria perianthomega (Vell.) Sleumer $\bullet \mathbf{\Delta}$ & tree & Lordêlo, R.P. 56 & AM, CAA, CER, MA \\
\hline Ximenia coriacea Engl. & tree & $\begin{array}{c}\text { Souza, J.S. } \\
\text { (HURB 20462) }\end{array}$ & CAA, CER \\
\hline \multicolumn{4}{|l|}{ Oleaceae } \\
\hline Chionanthus crassifolia (Mart.) P.S. Green & tree & $\begin{array}{c}\text { Souza, J.S. } \\
\text { (HURB 20488) }\end{array}$ & CAA, CER, MA \\
\hline
\end{tabular}


Table 1 (continuation)

\begin{tabular}{|c|c|c|c|}
\hline Family & Habit & Colector/Number & Phytogeographical Domain \\
\hline \multicolumn{4}{|l|}{ Orchidaceae } \\
\hline $\begin{array}{l}\text { Cattleya amethystoglossa Linden \& Rchb.f. } \\
\text { - } \Delta\end{array}$ & epi & Lordêlo, R.P. 5743 & CAA, MA \\
\hline Cyclopogon elatus (Sw.) Schltr. & herb & Moreira, D.M. 68 & CER, MA \\
\hline Dimerandra emarginata (G.Mey.) Hoehne & epi & Moreira, D.M. 270 & AM, MA \\
\hline Habenaria hamata Barb.Rodr. & herb & Moreira, D.M. 210 & AM, CAA, CER \\
\hline Lockhartia lunifera (Lindl.) Rchb.f. & epi & Moreira, D.M. 271 & AM, CER, MAA \\
\hline Miltonia flavescens (Lindl.) Lindl. • $\boldsymbol{\Delta}$ & epi & Lordêlo, R.P. 57-714 & CAA, CER, MA \\
\hline sp. 1 & epi & Costa, G. 1529 & $*$ \\
\hline \multicolumn{4}{|l|}{ Oxalidaceae } \\
\hline Oxalis puberula Nees \& Mart. & herb & Moreira, D.M. 76 & CER, MA \\
\hline \multicolumn{4}{|l|}{ Passifloraceae } \\
\hline Passiflora amethystina J.C.Mikan & vine & Moreira, D.M. 228 & AM, CAA, CER, MA, PAN \\
\hline Passiflora foetida L. & vine & Moreira, D.M. 22 & $\begin{array}{l}\text { AM, CAA, CER, MA, PAN, } \\
\text { PAM }\end{array}$ \\
\hline \multicolumn{4}{|l|}{ Piperaceae } \\
\hline Piper aduncum L. & $*$ & Moreira, D.M. 20 & $\begin{array}{l}\text { AM, CAA, CER, MA, PAN, } \\
\text { PAM }\end{array}$ \\
\hline Piper amalago $\mathrm{L}$. & tree & $\begin{array}{c}\text { Souza, J.S. } \\
\text { (HURB 20506) }\end{array}$ & AM, CAA, CER, MA, PAN \\
\hline Piper corcovadensis (Miq.) C.DC. & shrub & Moreira, D.M. 218 & AM, MA \\
\hline Piper gaudichaudianum Kunth & $*$ & Costa, G. 1551 & AM, CER, MA \\
\hline Piper hispidum Sw. & shrub & Moreira, D.M. 16 & AM, CER, MA \\
\hline Piper sp. 1 & $*$ & Costa, G. 1546 & $*$ \\
\hline Piper umbellatum $\mathrm{L}$. & shrub & Moreira, D.M. 8 & AM, CER, MA \\
\hline \multicolumn{4}{|l|}{ Plantaginaceae } \\
\hline Angelonia salicariifolia Bonpl. & $* *$ & Conceição, S.F. 801 & CAA, CER, PAN \\
\hline sp. 1 & sub & Moreira, D.M. 133 & $*$ \\
\hline \multicolumn{4}{|l|}{ Poaceae } \\
\hline Acroceras zizanioides (Kunth) Dandy $\bullet \boldsymbol{\Delta}$ & herb & Lordêlo, R.P. 52-142 & AM, CAA, CER, MA \\
\hline Ichnanthus hirtus (Raddi) Chase • $\boldsymbol{\Delta}$ & herb & Pinto, G.C.P. 530 & MA \\
\hline Ichnanthus leiocarpus (Spreng.) Kunth $\bullet \boldsymbol{\Delta}$ & herb & Lordêlo, R.P. 56-92 & CAA, CER, MA \\
\hline Lasiacis divaricata (L.) Hitchc. & sub & Moreira, D.M. 98 & CAA, CER, MA \\
\hline Olyra ciliatifolia Raddi $\bullet \Delta$ & herb & Oliveira, R.P. 913 & AM, CAA, CER, MA, PAN \\
\hline Olyra latifolia $\mathrm{L}$. & herb & Moreira, D.M. 90 & AM, CAA, CER, MA, PAN \\
\hline Pharus latifolius L. &.. & Oliveira, R.P. 912 & AM, CAA, MA \\
\hline Raddia guianensis (Brongn.) Hitchc. & herb & Oliveira, R.P. 911 & AM, MA \\
\hline \multicolumn{4}{|l|}{ Polygalaceae } \\
\hline Asemeia sp. & shrub & Moreira, D.M. 244 & * \\
\hline $\begin{array}{l}\text { Caamembeca grandifolia (A.St.-Hil. \& } \\
\text { Moq.) J.F.B.Pastore }\end{array}$ & shrub & Moreira, D.M. 264 & MA \\
\hline Polygala paniculata L. & herb & Moreira, D.M. 96 & AM, CAA, CER, MA, PAM \\
\hline
\end{tabular}


Table 1 (continuation)

\begin{tabular}{|c|c|c|c|}
\hline Family & Habit & Colector/Number & Phytogeographical Domain \\
\hline \multicolumn{4}{|l|}{ Polygonaceae } \\
\hline Coccoloba alnifolia Casar. & tree & $\begin{array}{l}\text { Souza, J.S. } \\
\text { (HURB 20458) }\end{array}$ & CAA, MA \\
\hline Coccoloba declinata (Vell.) Mart & tree & $\begin{array}{c}\text { Souza, J.S. } \\
\text { (HURB 20459) }\end{array}$ & AM, CAA, CER, MA \\
\hline Coccoloba arborescens (Vell.) R.A.Howard. & tree & $\begin{array}{c}\text { Souza, J.S. } \\
\text { (HURB 20498) }\end{array}$ & AM, CER, MA \\
\hline Coccoloba mollis Casar. & tree & Moreira, D.M. 2 & AM, CAA, CER, MA \\
\hline \multicolumn{4}{|l|}{ Primulaceae } \\
\hline Clavija caloneura Mart. & sub & Moreira, D.M. 37 & MA \\
\hline \multicolumn{4}{|l|}{ Rubiaceae } \\
\hline Borreria humifusa Mart. & $*$ & Costa, G. 1552 & MA \\
\hline $\begin{array}{l}\text { Borreria ocymifolia (Roem. \& Schult.) } \\
\text { Bacigalupo \& E.L.Cabral }\end{array}$ & sub & Moreira, D.M. 1052 & AM, CAA, CER, MA \\
\hline Chomelia anisomeris Müll.Arg. & shrub & Costa, G. 1534 & AM, CAA, CER, MA \\
\hline Chomelia martiana Müll.Arg. & tree & Moreira, D.M. 110 & CAA, CER, MA \\
\hline Chomelia obtusa Cham. \& Schltdl. & shrub & Moreira, D.M. 84 & AM, CAA, CER, MA \\
\hline Chomelia sericea Müll.Arg. & tree & $\begin{array}{l}\text { Souza, J.S. } \\
\text { (HURB 20449) }\end{array}$ & CAA, CER \\
\hline Chomelia sp1. & shrub & Moreira, D.M. 9 & $*$ \\
\hline Coccocypselum cordifolium Nees \& Mart. & $*$ & Costa, G. 1552 & AM, CAA, CER, MA \\
\hline Coussarea leptopus Müll.Arg. $\mathbf{\Delta}$ & shrub & Gusmão, E.F. 407 & MA \\
\hline Faramea axilliflora DC. & shrub & Moreira, D.M. 48 & $\begin{array}{c}\text { AM, CAA, CER, MA, PAN, } \\
\text { PAM }\end{array}$ \\
\hline Faramea hyacinthina Mart. $\mathbf{\Delta} \Delta$ & shrub & Moreira, D.M. 145 & MA \\
\hline Faramea oligantha Müll.Arg. & shrub & Moreira, D.M. 190 & MA \\
\hline Gonzalagunia dicocca Cham. \& Schltdl. $\mathbf{\Delta}$ & shrub & Costa, G. 1559 & AM, CAA, CER, MA \\
\hline Hamelia patens Jacq. $\mathbf{\square}$ & shrub & Gusmão, E.F. 410 & $\begin{array}{c}\text { AM, CAA, CER, MA, PAN, } \\
\text { PAM }\end{array}$ \\
\hline Ixora muelleri (Muell. Arg.) Bremekamp $\mathbf{\square}$ & shrub & Gusmão, E.F. 403 & MA \\
\hline Machaonia acuminata Bonpl. & tree & Souza, J.S. 20501 & CAA, CER, MA \\
\hline $\begin{array}{l}\text { Margaritopsis chaenotricha (DC.) } \\
\text { C.M.Taylor } \mathbf{\Delta}\end{array}$ & shrub & Moreira, D.M. 26 & MA \\
\hline Mitracarpus hirtus (L.) DC. & herb & Costa, G. 1537 & AM, CAA, CER \\
\hline Palicourea racemosa (Aubl.) Borhidi & shrub & Moreira, D.M. 129 & AM, CER, MA \\
\hline Posoqueria latifolia (Rudge) Schult. & shrub & Moreira, D.M. 70 & AM, CAA, CER, MA \\
\hline Psychotria carthagenensis Jacq. $\mathbf{\Delta}$ & shrub & Gusmão, E.F. 406 & $\begin{array}{c}\text { AM, CAA, CER, MA, PAN, } \\
\text { PAM }\end{array}$ \\
\hline $\begin{array}{l}\text { Psychotria colorata (Willd. ex Schult.) Müll. } \\
\text { Arg. }\end{array}$ & herb & Moreira, D.M. 208 & AM, CER, MA \\
\hline Psychotria cupularis (Müll.Arg.) Standl. & shrub & Moreira, D.M. 69 & AM, MA \\
\hline Psychotria deflexa DC. $\bullet \mathbf{\square}$ & shrub & Moreira, D.M. 14 & AM, CAA, CER, MA \\
\hline
\end{tabular}


Table 1 (continuation)

\begin{tabular}{|c|c|c|c|}
\hline Family & Habit & Colector/Number & Phytogeographical Domain \\
\hline Randia armata (Sw.) DC & tree & $\begin{array}{c}\text { Souza, J.S. } \\
\text { (HURB 20486) }\end{array}$ & AM, CER, MA \\
\hline Randia calycina Cham. & tree & Moreira, D.M. 165 & AM, CAA, CER, MA \\
\hline Rudgea aff. erythrocarpa Müll. Arg. & tree & Moreira, D.M. 166 & MA \\
\hline Rudgea jacobinensis Müll.Arg. $\mathbf{\square}$ & shrub & Gusmão, E.F. 405 & MA, CAA \\
\hline Tocoyena bullata (Vell.) Mart. & tree & $\begin{array}{l}\text { Souza, J.S. } \\
\text { (HURB 20505) }\end{array}$ & CAA, CER, MA \\
\hline sp. 1 & vine & Moreira, D.M. 44 & * \\
\hline \multicolumn{4}{|l|}{ Rutaceae } \\
\hline $\begin{array}{l}\text { Angostura bracteata (Nees \& Mart.) } \\
\text { Kallunki }\end{array}$ & shrub & Moreira, D.M. 158 & MA \\
\hline Citrus sp. & shrub & Moreira, D.M. 275 & EXOTIC \\
\hline Conchocarpus macrophyllus J.C.Mikan & shrub & Moreira, D.M. 147 & MA \\
\hline Ertela trifolia (L.) Kuntze & herb & Moreira, D.M. 172 & AM, CAA, CER, MA \\
\hline Erythrochiton brasiliensis Nees \& Mart. & shrub & Moreira, D.M. 182 & AM, MA \\
\hline Esenbekia sp 1 & sub & Moreira, D.M. 71 & $*$ \\
\hline Pilocarpus spicatus A.St.-Hil. & herb & Moreira, D.M. 45 & CAA, CER, MA \\
\hline Metodrorea sp. & shrub & Moreira, D.M. 198 & * \\
\hline sp. 2 & $*$ & Moreira, D.M. 40 & * \\
\hline \multicolumn{4}{|l|}{ Salicaceae } \\
\hline Banara serrata (Vell.) Warb. & shrub & Moreira, D.M. 80 & AM, MA \\
\hline Casearia javitensis Kunth & tree & $\begin{array}{l}\text { Souza, J.S. } \\
\text { (HURB 20512) }\end{array}$ & AM, CAA, CER, MA \\
\hline Casearia sylvestris $\mathrm{Sw}$. & shrub & Moreira, D.M. 249 & AM, CAA, CER, PAN, PAM \\
\hline \multicolumn{4}{|l|}{ Sapindaceae } \\
\hline $\begin{array}{l}\text { Allophylus edulis (A.St.-Hil. et al.) Hieron. } \\
\text { ex Niederl. }\end{array}$ & tree & $\begin{array}{l}\text { Souza, J.S. } \\
\text { (HURB 20511) }\end{array}$ & AM, CAA, CER, PAN \\
\hline Allophylus racemosus $\mathrm{Sw}$. & tree & $\begin{array}{c}\text { Souza, J.S. } \\
\text { (HURB 20467) }\end{array}$ & AM, CAA, CER, MA \\
\hline Cupania oblongifolia Mart. & tree & Moreira, D.M. 12 & AM, CAA, CER, MA \\
\hline Matayba guianensis Aubl. & tree & $\begin{array}{c}\text { Souza, J.S. } \\
\text { (HURB 20508) }\end{array}$ & AM, CAA, CER, MA, PAN \\
\hline Paullinia elegans Cambess. & vine & Moreira, D.M. 85 & AM, CAA, CER, MA, PAN \\
\hline Paullinia revoluta Radlk. & shrub & Moreira, D.M. 109 & MA \\
\hline Paullinia rubiginosa Cambess. & vine & Moreira, D.M. 245 & AM, CER, MA \\
\hline Serjania pernambucensis Radlk. & vine & Costa, G. 1533 & CAA, MA \\
\hline sp. 1 & vine & Moreira, D.M. 142 & * \\
\hline sp. 2 & vine & Moreira, D.M. 86 & $*$ \\
\hline \multicolumn{4}{|l|}{ Sapotaceae } \\
\hline Manilkara salzmannii (A.DC.) H.J.Lam & tree & $\begin{array}{l}\text { Souza, J.S. } \\
\text { (HURB 20493) }\end{array}$ & CAA, MA \\
\hline Pouteria gardneri (Mart. \& Miq.) Baehni & tree & $\begin{array}{c}\text { Souza, J.S. } \\
\text { (HURB 20453) }\end{array}$ & AM, CAA, CER, MA \\
\hline
\end{tabular}


Table 1 (continuation)

\begin{tabular}{|c|c|c|c|}
\hline Family & Habit & Colector/Number & Phytogeographical Domain \\
\hline Pouteria gardneriana (A.DC.) Radlk. & tree & $\begin{array}{c}\text { Souza, J.S. } \\
\text { (HURB 20502) }\end{array}$ & CAA, CER, MA \\
\hline \multicolumn{4}{|l|}{ Smilacaceae } \\
\hline Smilax cissoides Mart. ex Griseb. & vine & Moreira, D.M. 67 & AM, CAA, CER, MA \\
\hline \multicolumn{4}{|l|}{ Solanaceae } \\
\hline Cestrum nocturnum L. & shrub & Conceição, S.F. 795 & $*$ \\
\hline Solanum americanum Mill. & herb & Moreira, D.M. 226 & $\begin{array}{c}\text { AM, CAA, CER, MA, PAM, } \\
\text { PAN }\end{array}$ \\
\hline Solanum maranguapense Bitter $\bullet \boldsymbol{\Delta}$ & shrub & Lordêlo, R.P. 5751 & MA \\
\hline Solanum paniculatum L. & sub & Moreira, D.M. 115 & AM, CAA, CER, MA \\
\hline Solanum polytrichum Moric. & arb & Costa, G. 1050 & CAA, MA \\
\hline Solanum pseudoquina A. St.-Hil. & tree & $\begin{array}{c}\text { Souza, J.S. } \\
\text { (HURB 20491) }\end{array}$ & MA \\
\hline \multicolumn{4}{|l|}{ Urticaceae } \\
\hline Cecropia hololeuca Miq. & tree & Moreira, D.M. 272 & CER, MA \\
\hline Cecropia pachystachya Trécul & tree & Moreira, D.M. 273 & AM, CAA, CER, MA, PAN \\
\hline sp. 1 & shrub & Moreira, D.M. 194 & $*$ \\
\hline \multicolumn{4}{|l|}{ Verbenaceae } \\
\hline Lantana canescens Kunth & $*$ & Costa, G. 1538 & AM, CAA, CER, MA \\
\hline Lantana $\mathrm{sp}$ & sub & Moreira, D.M. 114 & $*$ \\
\hline Lippia sp. & shrub & Conceição, S.F. 813 & $*$ \\
\hline Priva lappulacea (L.) Pers. & herb & Moreira, D.M. 82 & AM, CER, MA, PAN \\
\hline Tamonea spicata Aubl. & $*$ & Costa, G. 1550 & CAA, CER, MA \\
\hline \multicolumn{4}{|l|}{ Violaceae } \\
\hline Amphirrhox longifolia (A.St.-Hil.) Spreng. & tree & Moreira, D.M. 184 & AM, MA \\
\hline
\end{tabular}

\section{Discussion}

In general, the Atlantic Forest (AF) is marked by the predominance of some groups with a high number of species, such as Orchidaceae, Fabaceae, Myrtaceae, Rubiaceae, Melastomataceae and Bromeliaceae (Amorim et al. 2005, Amorim et al. 2009, Thomas et al. 2009, Oliveira et al. 2016) (figures 4-5). The Sapindaceae, Acanthaceae and Araceae are not usually the ones with the highest number of species in floristic surveys for AF (Amorim et al. 2005, Amorim et al. 2009 Thomas et al. 2009, Coelho \& Amorim 2014). The differences between the main families found in this study and other floristic surveys in the Atlantic Forest of Bahia may be associated to the different degree of conservation of the studied areas and the different interactions among physical, biological, geological and altitude factors. Such factors can cause floristic variations in the family-level communities and lower taxonomic categories (Lingner et al. 2015).
The representative number of species of the Sapindaceae family in this study is mainly due to the occurrence of a significant number of vines species (table 1). Of the 10 species reported for this group, five are vines plants, which may indicate that Serra da Copioba has a secondary successional state, since this habit in ombrophilous forests is commonly associated with border areas and altered vegetation (Engel et al. 1998, Pereira et al. 2016). The Acanthaceae family is one of the most important families in the number of species (10 spp.), Because seven species of the species reported here are endemic to the $\mathrm{AF}$, and three of them are endemic to Bahia (Flora do Brasil under construction 2018). Some reproductive and dispersal characteristics of this family such as showy and bisexual flowers and fruits with explosive dehiscence (Souza \& Lorenzi 2012, Braz \& Azevedo 2016), may have been fundamental for its success in Serra da Copioba, since forests altered species tend to have fewer dispersers, negatively affecting zoochoric species (Volpato et al. 2018). 
The richness found for Araceae (eight spp.) may be associated with the previous survey of the family made by Andrade et al. (2007) for the study area. Of the eight species listed here, four (Anthurium pentaphyllum (Aubl.) G.Don, Heteropsis oblongifolia Kunth, Monstera adansonii Schott and Philodendron pedatum (Hook.) Kunth) were collected by Andrade et al. 2007. In addition, the species richness can be linked to a variety of biotic to abiotic factors and may also be associated with sampling effort (Gaston 2000, Felfili et al. 2011, Arceo-Gómez et al. 2018). Anthurium radicans K. Koch \& Haage, a vulnerable species (Coelho et al. 2014), has increased its extent of occurrence, however, occurring in small and unique populations that are threatened by agricultural activities in Serra da Copioba (figure 4).

The richness of Rubiaceae, Fabaceae, Melastomataceae and Myrtaceae has been reported in studies on areas of AF of Bahia, regardless of location and phytophisionomy (Amorim et al. 2005, Amorim et al. 2009, Thomas et al. 2009).

Myrtaceae has been pointed out by several authors as indicator of forest quality, where there is a direct relation between fall in family richness and habitat reduction (Rodal et al. 2005, Amorim et al. 2009, Rigueira et al. 2013). The Serra da Copioba shelters areas at different levels of anthropization, less altered areas conserve relative diversity of Myrtaceae, which may be related to the fact that the species of this family does not produce valuable woods (Fontana et al. 2014), and thus not be targets of illegal extraction. Both this activity and livestock and agriculture devastated the native vegetation of the Recôncavo da Bahia region, and still threaten the few fragments of Atlantic Forest that remained in the region (Nardi 2013). Melastomataceae is a typical group found in forests in a recent secondary state, mainly because they are heliophiles and occupy clearings, forming a fundamental group in areas of recovery and forest regeneration in the AF (Melo Junior et al. 2017, Pessoa et al. 2012).

Most threatened groups, such as Orchidaceae, preferentially occupy primary forests with high conservation status and are sensitive to climate change, forest fragmentation and soil pollution (Fajardo et al. 2016). Therefore, the most conserved forest areas such as those found in southern Bahia tend to have a higher concentration of Orchidaceae species and areas that are more vulnerable to anthropic action, such as Serra da Copioba, tend to have a reduced number of species (seven species, figure 2).

The number of families found in this study ( 77 families) was similar to the one obtained in the Serra da Jiboia survey (between Santa Teresinha and Castro Alves, Sobrinho \& Queiroz (2005), which presented 80 families. However, they were lower than the values found by Amorim et al. (2009) in the municipalities of Barro Preto (87), Arataca (110) and Camacan (103) (table 2). This fact is due to the high degree of conservation that these forests are in the South of the State. Values higher than those found by Thomas et al. (2009) in Jussari (56 families) (table 2), all located in the South of Bahia that despite being an area with a high degree of conservation associated with other factors such as, the sampling that was done through plot methods and inclusion of plants with DAP of $\geq 5 \mathrm{~cm}$, excludes most of the herbs, and the temporal effort of collection did not comprise a large time interval, which can result in lower values of wealth (Freitas \& Magalhães 2012).

The proportion between arboreal and non-arboreal habits reveals a greater richness of this in Serra da Copioba vegetation (figure 3 ). The variation in the proportion of non-arboreal / arboreal habits in the several surveys for the Atlantic Forest in the State of Bahia has already been reported (Amorim et al. 2005, Amorim et al. 2009, Thomas et al. 2009). However, the proportion of non-arboreal species was only higher than the tree species in the Reserva Particular do Patrimônio Natural Serra Bonita (Amorim et al. 2009). This relationship needs to be better investigated, as it may reflect better conservation status or variations in slope, topography, latitude, altitude in the studied environments (Coelho \& Amorim 2014).

The epiphytic component in Serra da Copioba was inferior when compared to the number of other species of Atlantic Forest in Bahia (Amorim et al. 2005, Amorim et al. 2009, Thomas et al. 2009, Coelho \& Amorim 2014). This type of habit has been shown to be an important indicator of well-conserved forests and has a relevant ecological role, as epiphytes influence nutrient cycles and provide shelter and feed (Freitas et al. 2016, Leitman et al. 2015). Vine habit is commonly found in almost all forest communities. Despite this, it is more common in forests with higher levels of degradation and forest edges due to clearings and consequent high luminosity. The low number of vines species found in Serra da Copioba may be due to the high slope and altitude of the area, which are possible limiting factors for the volatiles / lianas / creepers, which prefer lower and flat lands (Engel et al. 1998).

Regarding the exotic species, we can infer that the presence of individuals of Artocarpus heterophyllus Lam. (jackfruit tree) in the interior of Serra da Copioba has caused concern, since it has already been observed that this species interferes in species richness, diversity and soil composition, being these strong threats to fragments of AF (Fabricante et al. 2012). Species of Cordyline sp. have been reported as invasive in the Cerrado (Horowits et al. 2013), as well as species of Citrus sp. were already evidenced as invasive potentials in the AF (MMA 2006).

The advanced level of anthropization and the still recurrent threats such as the removal of wood, suppression of vegetation for pasture replacement and the presence of domestic animals (cattle and horses) within the Serra da Copioba area have rendered this fragment fragile, endangering species that occur there. This factor can be evaluated through analysis of historical collections. 


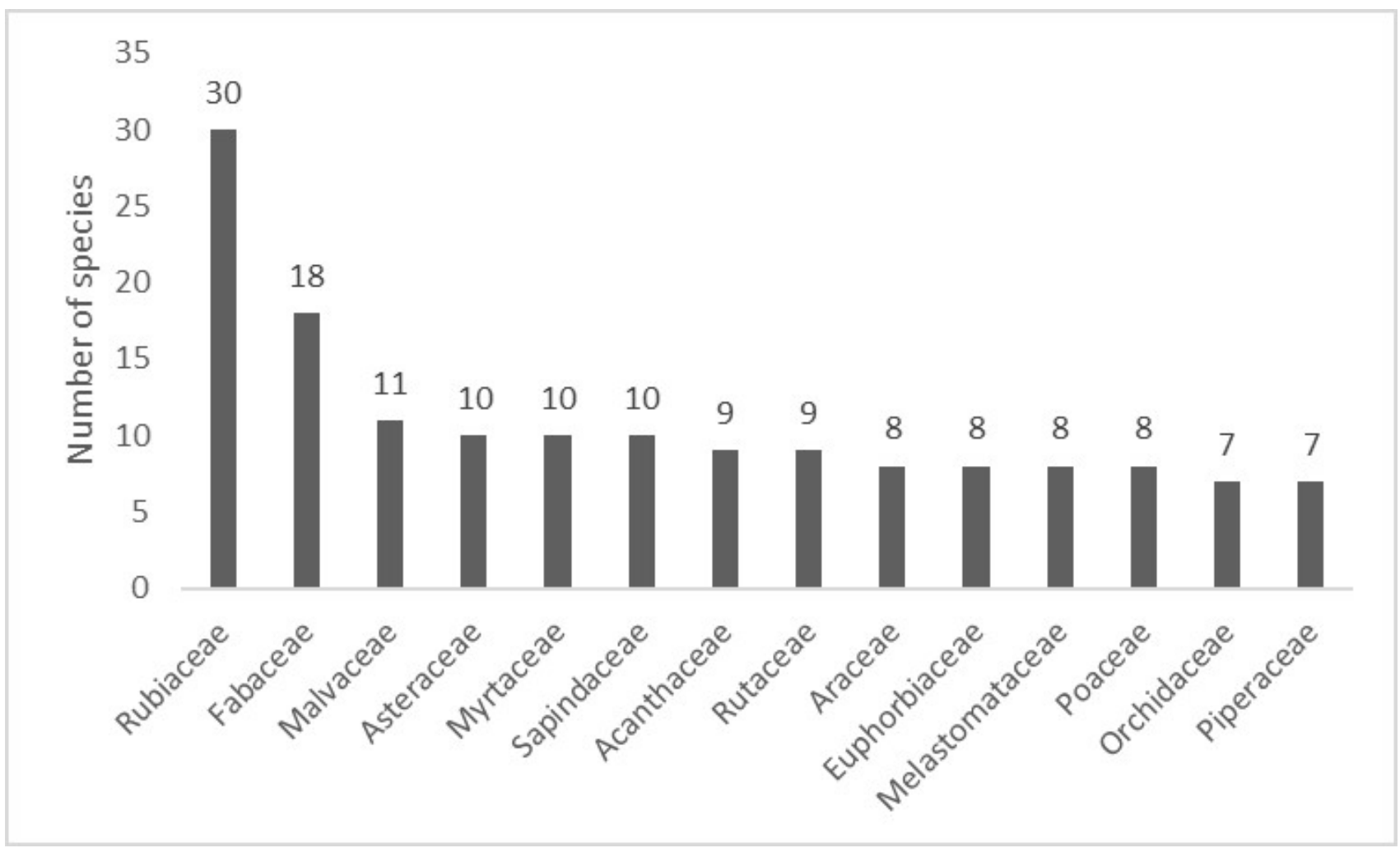

Figure 2: Families with the highest number of species in Serra da Copioba, São Felipe, Bahia State, Brazil.

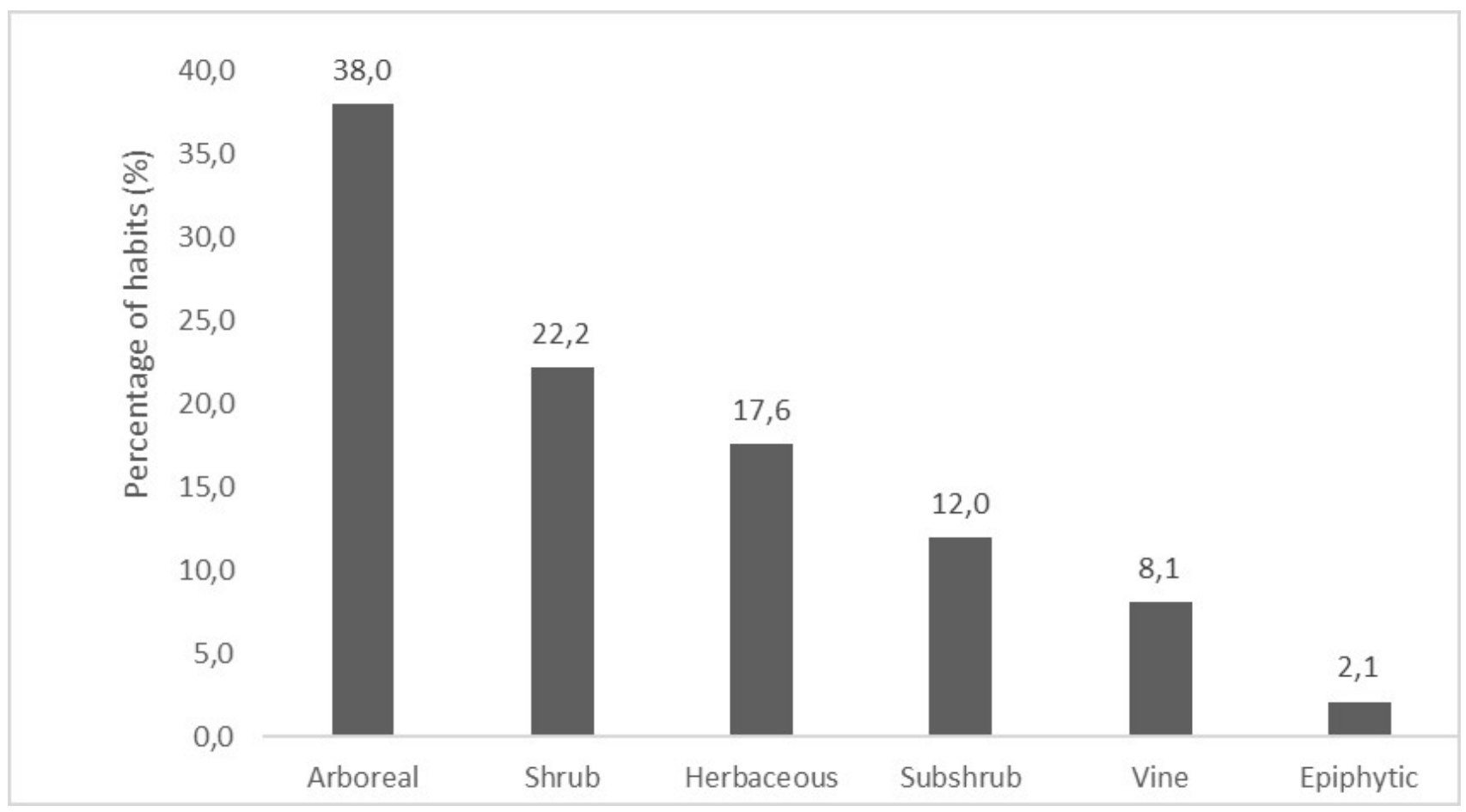

Figure 3: Percentage of habits found in the angiosperms occurring in Serra da Copioba, São Felipe, Bahia State, Brazil. 

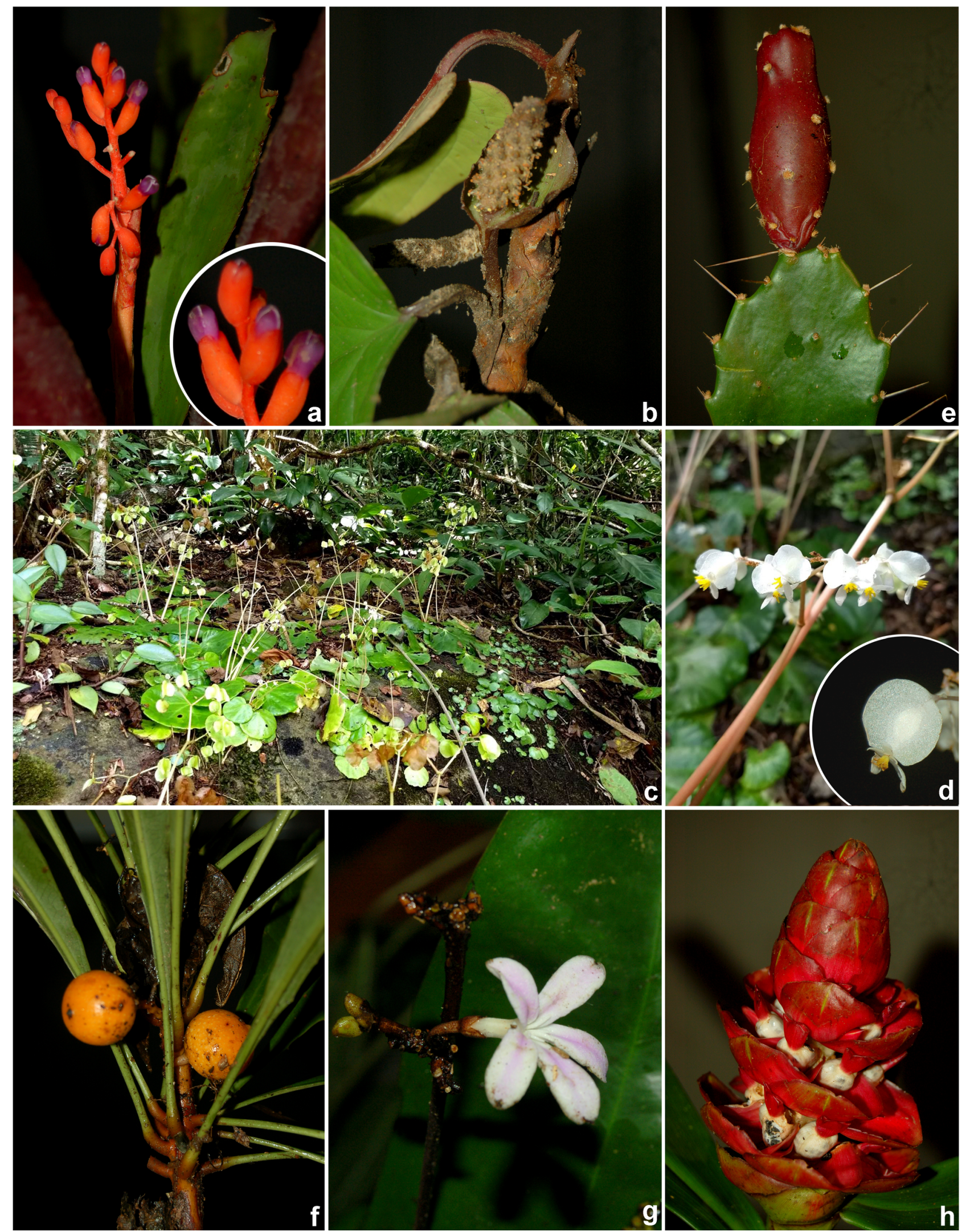

Figure 4. Species of angiosperms occurring in Serra da Copioba, São Felipe, Bahia State, Brazil. a. Aechmea fulgens Brongn. b. Anthurium radicans K.Koch \& Haage. c-d. Begonia delicata Gregório \& J.A.S. Costa. e. Brasiliopuntia brasiliensis (Willd.) A.Berger. f. Clavija caloneura Mart. g. Conchocarpus macrophyllus J.C.Mikan. h. Costus scaber Ruiz \& Pav. 


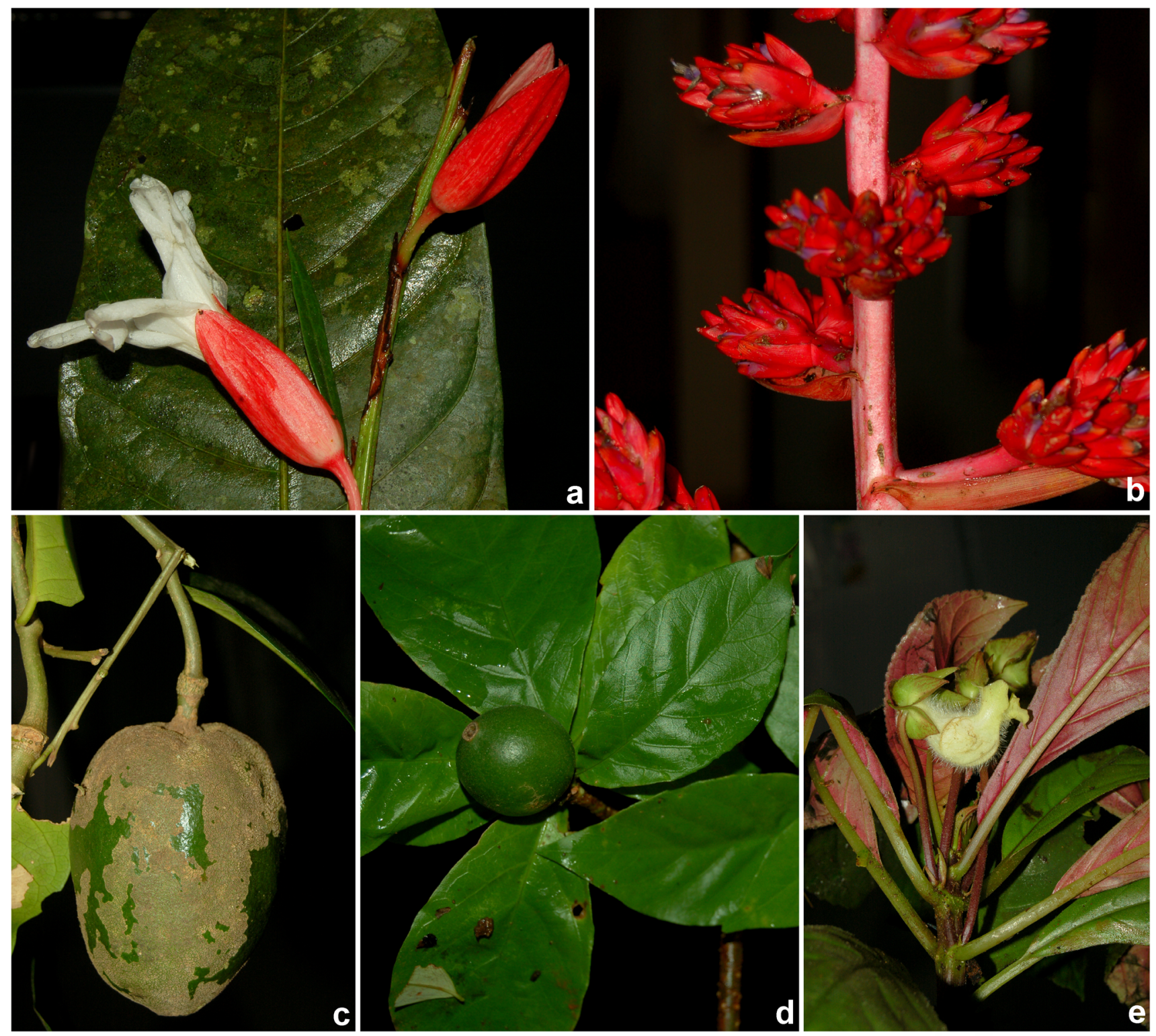

Figure 5. Species occurring in Serra da Copioba, São Felipe, Bahia State, Brazil. a. Erythrochiton brasiliensis Nees \& Mart. b. Hohenbergia stellata Schult. \& Schult.f. c. Neocalyptrocalyx grandipetala (Maguire \& Steyerm.) Cornejo \& Iltis. d. Randia calycina Cham. e. Sinningia barbata (Nees \& Mart.) G.Nicholson.

Of the 36 species from historical collections, 24 were not found again in Serra da Copioba, for example, Cattleya amethystoglossa Linden \& Rchb.f. (Orchidaceae) that is threatened with extinction and Heteropterys sanctorum W.R.Anderson (Malpighiaceae) that is critically endangered (BFG 2015). In most cases, the risk of extinction or disappearance of species is related to human activities and unsustainable use of natural resources, which culminate in the degradation of habitats and the disappearance of species (Penedo et al. 2015, Srbek-Araujo et al. 2017). The only species that were sampled at the three intervals (decades of 50, 70 and the present) are species of wide distribution, as for example, Psychotria deflexa DC. (Rubiaceae) which has distribution in several biomes such as Caatinga, Cerrado, Amazon and Atlantic Forest (BFG 2015), and Bomarea edulis (Tussac) Herb. (Alstromeriaceae), typical of forest edges (Lorenzzi \& Souza, 2012). 
Table 2: Comparison of the main families and number of species in surveys in areas of Atlantic Forest in the State of Bahia, Brazil.

\begin{tabular}{|c|c|c|c|c|c|}
\hline Municipality & $\begin{array}{l}\text { Species } \\
\text { Number }\end{array}$ & $\begin{array}{l}\text { Family } \\
\text { number }\end{array}$ & Main families & Method & Reference \\
\hline São Felipe & 306 & 77 & $\begin{array}{c}\text { Rubiaceae, Fabaceae, Acanthaceae, } \\
\text { Asteraceae, Malvaceae, Myrtaceae and } \\
\text { Sapindaceae }\end{array}$ & Floristic & Present study \\
\hline Jussari & 264 & 56 & $\begin{array}{c}\text { Fabaceae, Myrtaceae, Sapotaceae, Meliaceae, } \\
\text { Moraceae, Lauraceae and Rubiaceae }\end{array}$ & $\begin{array}{l}\text { Plot/Inclusion } \\
\text { criteria } \geq 5 \mathrm{~cm}\end{array}$ & $\begin{array}{l}\text { Thomas et al. } \\
\text { (2009) }\end{array}$ \\
\hline Barro Preto & 412 & 87 & $\begin{array}{c}\text { Orchidaceae, Rubiaceae, Bromeliaceae, } \\
\text { Melastomataceae, Poaceae, Piperaceae and } \\
\text { Araceae }\end{array}$ & Floristic & $\begin{array}{l}\text { Amorim et al. } \\
\quad(2009)\end{array}$ \\
\hline Arataca & 709 & 110 & $\begin{array}{c}\text { Orchidaceae, Melastomataceae, Bromeliaceae, } \\
\text { Asteraceae, Myrtaceae, Fabaceae and } \\
\text { Piperaceae }\end{array}$ & Floristic & $\begin{array}{l}\text { Amorim et al. } \\
\quad(2009)\end{array}$ \\
\hline Camacan & 628 & 103 & $\begin{array}{l}\text { Orchidaceae, Rubiaceae, Melastomataceae, } \\
\text { Asteraceae, Poaceae, Solanaceae and Fabaceae }\end{array}$ & Floristic & $\begin{array}{l}\text { Amorim et al. } \\
\quad(2009)\end{array}$ \\
\hline $\begin{array}{c}\text { Santa } \\
\text { Terezinha/ } \\
\text { Castro Alves }\end{array}$ & 269 & 80 & $\begin{array}{c}\text { Fabaceae, Rubiaceae, Asteraceae, } \\
\text { Melastomataceae, Orchidaceae, Myrtaceae and } \\
\text { Solanaceae }\end{array}$ & Floristic & $\begin{array}{c}\text { Coelho \& } \\
\text { Amorim (2005) }\end{array}$ \\
\hline
\end{tabular}

Despite the advanced degree of devastation in Serra da Copioba, the area is still an important representative of the local flora and has considerable species richness, as indicated by the results obtained here (table 1). This area comprises several important species such as Eugenia expansa Spring ex Mart. (Myrtaceae), species that was first recorded by this study for the State of Bahia and Begonia delicata Gregório \& J.A.S. Costa (Begoniaceae), a recently described species (2015), micro-endemic to the study area, found only in the highest areas (above $250 \mathrm{~m}$ altitude) and well conserved in the Serra da Copioba (figure 4).

In this way, the surroundings of Serra da Copioba, as well as every local community, need to be made aware of the importance of conservation of this fragment. The threats of the Atlantic Forest evidenced by the literature, in addition to the fact that the species found in the 50s and 70s have not been recovered, show a high degree of atrocities in Serra da Copioba. This fact can also be verified by the observation of the increasing deforestation that continues towards the summit during the collection years of this study, where the endemic species (Begonia delicata) of Serra da Copioba occurs. There is no doubt about the necessity and urgency of using protective measures around the Serra da Copioba, as inspection to prevent deforestation as well as actions to raise awareness of the local population.

\section{Acknowledgments}

The authors are grateful to the following taxonomists who helped in the species identification: K. Pimenta (Araceae); M. Alves and G.V. Fonseca (Asteraceae); B.
Gregório (Begoniaceae); E.H. Souza (Bromeliaceae); L.C. Marinho (Clusiaceae); T. Flores (Meliaceae); A. Machado (Moraceae); A. Stadnik (Myrtaceae); C. Silva (Poaceae); W.O. Fonseca and J. Jardim (Rubiaceae) and M.L.S. Guedes (several families). This paper is part of DMM MSc Dissertation and this study was financed in part by the Coordenação de Aperfeiçoamento de Pessoal de Nível Superior - Brasil (CAPES) - Finance Code 001. LYSA acknowledges support from the Fundação de Amparo à Pesquisa da Bahia (FAPESB) (grants RED0034/2014).

\section{Literature cited}

Amorim, A.M., Jardim, J.G., Clifton, B.C., Fiaschi, P., Thomas, W.W. \& Carvalho, A.M.V. 2005. The vascular plants of a forest fragment in southern Bahia, Brazil. Sida, Contributions to Botany 21: 1726-1752.

Amorim, A.M., Jardim, J.G., Lopes, M.M.M., Fiaschi, P., Borges, R.A.X., Perdiz, R.O. \& Thomas, W.W. 2009. Angiospermas em remanescentes de floresta montana no sul da Bahia, Brasil. Biota Neotropica 9: 313-348.

Andrade, I.M., Mayo, S.J., Van den Berg, C., Fay, M.F., Chester, M., Lexer, C. \& Kirkup, D. 2007. A preliminary study of genetic variation in populations of Monstera adansonii var. klotzchiana (Araceae) from North-East Brazil, estimated with AFLP molecular markers. Annals of Botany 100: 1143-1154.

APG IV. 2016. An update of the Angiosperm Phylogeny Group classification for the orders and families of flowering plants: APG IV. Botanical Journal of the Linnean Society 181: 1-20. 
Arceo-Gómez, G., Alonso, C., Ashman, T. \& Parra-Tabla, V. 2018. Variation in sampling effort affects the observed richness of plant-plant interactions via heterospecific pollen transfer: implications for interpretation of pollen transfer networks. American Journal of Botany 105: 1601-1608.

BFG. 2015. Growing knowledge: an overview of Seed Plant diversity in Brazil. Rodriguésia 66: 1085-1113.

Braz, D.M. \& Azevedo, I.H.F. 2016. Acanthaceae da Marambaia, Estado do Rio de Janeiro, Brasil. Hoehnea 43: 497-516.

Bridson, D. \& Forman, L. 2004. The Herbarium Handbook. The Royal Botanic Garden, Kew.

Carnaval, A.C. \& Moritz, C. 2008. Historical climate modelling predicts patterns of current biodiversity in the Brazilian Atlantic forest. Journal of Biogeography 35: 1187-1201.

Coelho, M.M. \& Amorim, A.M. 2014. Floristic composition of the Montane Forest in the Almadina - Barro Preto axis, Southern Bahia, Brazil. Biota Neotropica 14: 1-41.

CRIA. 2016. Centro de referência e informação ambiental. Disponível em http://www.cria.org.br (acesso em 20II-2018).

Engel, V.L., Fonseca, R.C.B. \& Oliveira, R.E. 1998. Ecologia de lianas e o manejo de fragmentos florestais. Série Técnica IPEF 12: 43-64.

Fabricante, J.R., Araújo, K.C.T., Andrade, L.A. \& Ferreira, J.V.A. 2012 Invasão biológica de Artocarpus heterophyllus Lam. (Moraceae) em um fragmento de Mata Atlântica no Nordeste do Brasil: impactos sobre a fitodiversidade e os solos dos sítios invadidos. Acta Botanica Brasilica 26: 399-407.

Fajardo, C.G., Vieira, F.A. \& Molina, W.F. 2016. Conservação Genética de Populações Naturais: Uma Revisão para Orchidaceae. Biota Amazônia 6: 108-118.

Felfili, J.M., Roitman, I., Medeiros, M.M. \& Sanchez, M. 2011. Procedimentos e métodos de amostragem da vegetação. In: J. M. Felfili, P.V. Eisenlohr, M.M.R.F. Melo, L. A. Andrade \& J.A.A.M. Neto (eds.). Fitossociologia no Brasil. UFV, Viçosa, pp. 86-121.

Flora do Brasil 2020 em construção. Jardim Botânico do Rio de Janeiro. Disponível em http://floradobrasil. jbrj.gov.br/reflora/floradobrasil/FB9079 (acesso em 29-I-2018).

Fontana, C., Gasper, A.L. \& Sevegnani, L. 2014. Espécies raras e comuns de Myrtaceae da Floresta Estacional Decidual de Santa Catarina, Brasil. Rodriguésia 65: 767-776.

Freitas, L., Salino, A., Menini Neto, L., aAlmeida, T.E., Mortara, S.R., Stehmann, J.R., Amorim, A.M., Guimarães, E.F., Coelho, M.N., Zanin A. \& Forzza, R.C. 2016. A comprehensive checklist of vascular epiphytes of the Atlantic Forest reveals outstanding endemic rates. PhytoKeys 58: 65-79.
Freitas, W.K. \& Magalhães, L.M.S. 2012. Métodos e Parâmetros para Estudo da Vegetação com Ênfase no Estrato Arbóreo. Floresta e Ambiente 19: 520-540.

Gaston, K.J. 2000. Global patterns in biodiversity. Nature 405: 200-227.

Gonçalves, E.G. \& Lorenzi, H. 2007. Morfologia vegetal: organografia e dicionário ilustrado de morfologia das plantas vasculares. Instituto Plantarum de Estudos da Flora, São Paulo.

Horowitz, C., Martins, C.R. \& Walter, B.M.T. 2013. Flora exótica no Parque Nacional de Brasília: levantamento e classificação das espécies. Biodiversidade Brasileira 3: $50-73$.

JBRJ. 2012. Instituto de Pesquisas Jardim Botânico do Rio de Janeiro. Jabot. - Banco de dados da floresta brasileira. Disponível em http://www.jbrj.gov.br/jabot (acesso em 20-II-2018)

Joly, C.A., Metzger, J.P. \& Tabarelli, M. 2014. Experiences from the Brazilian Atlantic Forest: ecological findings and conservation initiatives. New phytologist 204: 459473.

Leitman, P., Amorim, A.M., Sansevero, J.B. \& Forzza, R.C. 2015. Floristic patterns of the epiphytes in the Brazilian Atlantic Forest, a biodiversity hotspot. Botanical Journal of the Linnean Society 179: 587-601.

Lingner, D.V., Schorn, L.A., Sevegnani, L., Gasper, A.L., Meyer, L. \& Vibran, A.C. 2015. Floresta Ombrófila Densa De Santa Catarina - Brasil: Agrupamento e Ordenação Baseados em Amostragem Sistemática. Ciência Florestal 25: 933-946.

Melo Junior, J.C.F., Amorim, M.W., Arriola, I.A., Dirksen, J.S. \& Andreassi, F. 2017. Morphoanatomical adjustments of Tibouchina clavata (Melastomataceae) in ombrophilous forest and resting forest. Ciência e Natura 39: 484-493.

Mittermeier, R.A., Turner, W.R., Larsen, F.W., Brooks, T.M. \& Gascon C. 2011. Global Biodiversity Conservation: The Critical Role of Hotspots. In: Zachos, F.E. \& Habel, J.C. (Eds.). Biodiversity Hotspots: Distribution and Protection of Conservation Priority Areas, Berlin, pp. 3-22.

MMA. 2016. Ministério do Meio Ambiente. Espécies Exóticas Invasoras: Situação Brasileira. Secretaria de Biodiversidade e Florestas. Brasília.

Morellato, L.P.C. \& Haddad, C.F.B. 2000. Introduction: The Brazilian Atlantic Forest. Biotropica 32: 786-792.

Mori, S.A., Boom, B.A. \& Prance, G.T. 1981. Distribution patterns and conservation of eastern Brazilian coastal forest tree species. Brittonia 33: 233-245.

Mota, T.J.C.R., Carvalho, F.A., Ivanauskas, N.M. \& Eisenlohr, P.V. 2017. On the relevance of floristic and quantitative studies to the restoration of degraded areas: the case of the Atlantic Forest hotspot. Aims Environmental Science 4: 42-53. 
Nardi JB. 2013. Recôncavo Baiano: Entre Teorias e Práticas do Desenvolvimento Territorial. Olhares Sociais 2: 167192.

Newbold, T., Hudson, L.N., Hill, S.L.L., Contu, S., Lysenko, I., Senior, R.A., Börger, L., Bennett, D.J., Choimes, A., Collen, B., Day, J., De Palma, A., Díaz, S., Echeverria-Londoño, S., Edgar, M.J., Feldman, A., Garon, M., Harrison, M.L.K., Alhusseini, T., Ingram, D.J., Itescu, Y., Kattge, J., Kemp, V., Kirkpatrick, L., Kleyer, M., Laginha, P.C.D., Martin, C.D., Meiri, S., Novosolov, M., Pan, Y., Phillips, H.R.P., Purves, D.W., Robinson, A., Simpson, J., Tuck, S.L., Weiher, E., White, H.J., Ewers, R.M., Mace, G.M., Scharlemann, J.P.W., 9\& Purvis A. 2015. Global effects of land use on local terrestrial biodiversity. Nature 520: 45-50.

Oliveira, E.V.S., Gomes, L.A., Déda, R.M., Melo, L.M.S., Silva, A.C.C., Farias, M.C.V. \& Prata, A.P.N. 2016. Floristic survey of the Mata do Junco Wildlife Refuge, Capela, Sergipe State, Brazil. Hoehnea 43: 645-667.

Penedo, T.S.A., Moraes, M.A., Borges, A.X.B., Maurenza, D., Judice, D.M. \& Martinelli, G. 2015. Considerations on extinct species of Brazilian flora. Rodriguésia 66 : 711-715.

Pereira, L.A., Amorim, B.S., Alves, M., Somner, G.V. \& Barbosa, M.R.V. 2016. Flora da Usina São José, Igarassu, Pernambuco: Sapindaceae. Rodriguésia 67: 1047-1059.

Pessoa, M.S., Vleeschouwer, K.M., Talora, D.C., Rocha, L. \& Amorim, A.M.A. 2012. Reproductive phenology of Miconia mirabilis (Melastomataceae) within three distinct physiognomies of Atlantic Forest, Bahia, Brazil. Biota Neotropica 12: 49-56.

Rezende, C.L., Scarano, F.R., Assad, E.D., Joly, C.A., Metzger, J.P., Strassburg M., Tabarelli, M., Fonseca, G.A. \& Mittermeir, R.A. 2018. From hotspot to hopespot: An opportunity for the Brazilian Atlantic Forest. Perspectives in Ecology and Conservation 16: 208-214.
Rigueira, D.M.G., Rocha, P.L.B. \& Mariano-Neto, E. 2013. Forest cover, extinction thres holds and time lags in woody plants (Myrtaceae) in the Brazilian Atlantic Forest: resources for conservation. Biodiversity and Conservation 22: 3141-3163.

Rodal, M.J.N., Lucena, M.F.A., Andrade, K.V.S.A. \& Melo, A.L. 2005. Mata Do Toró: Uma Floresta Estacional Semi-Decidual De Terras Baixas Nordeste Do Brasil. Hoehnea 32: 1-12.

SEI. 2017. Superintendência de Estudos Econômicos e Sociais da Bahia. Disponível em www.sei.ba.gov.br (acesso em 15-X-2017).

Sobrinho, J.G.C. \& Queiroz, L.P. 2005. Composição florística de um fragmento de Mata Atlântica na Serra da Jibóia, Santa Terezinha, Bahia, Brasil. Sitientibus 5: 20-28.

SOS Mata Atlântica. 2016. Florestas: A Mata Atlântica. Disponível em https://www.sosma.org.br/nossa-causa/amata-atlantica/ (acesso em 15-VIII-2018).

Souza, V.C. \& Lorenzi, H. 2012. Botânica Sistemática: guia ilustrado para identificação das famílias de Fanerógamas nativas e exóticas no Brasil, baseado em APG III. 3 ed, Instituto Plantarum, Nova Odessa.

Srbek-Araujo, A.C., Gnocchi, A.P., Guimarães, L.J. \& Roper, J.J. 2017. Defaunation as a trigger for the additional loss of plant species in fragmented landscapes: considerations on the state of Espírito Santo, southeastern Brazil. Rodriguésia 68: 2001-2017.

Thomas, W.W., Jardim, J.J.G., Fiaschi, P., Neto, E.M. \& Amorim, A.A. 2009. Composição florística e estrutura do componente arbóreo de uma área transicional de Floresta Atlântica no sul da Bahia, Brasil. Brazilian Journal of Botany 32: 65-78.

Thomas, W.W., Forzza, R.C., Michelangeli, F.A., Giulietti, A.M. \& Leitman, P.M. 2012. Large-scale monographs and floras: the sum of local floristic research. Plant Ecology \& Diversity 5: 217-223.

Volpato, G.H., Miranda Neto, A. \& Martins, S.V. 2018. Avifauna como bioindicadora para avaliação da restauração florestal: estudo de caso em uma floresta restaurada com 40 anos em viçosa - MG. Ciência Florestal 28: 336-344. 